\title{
A New Class of Generalized Burr III Distribution for Lifetime Data
}

\section{Olobatuyi Kehinde, Asiribo Osebi, Dawodu Ganiyu}

Department of Statistics, Federal University of Agriculture, Abeokuta, Nigeria

Email address:

olobatuyi.kehindei@pg.funaab.edu.ng(O.Kehinde)

\section{To cite this article:}

Olobatuyi Kehinde, Asiribo Osebi, Dawodu Ganiyu. A New Class of Generalized Burr III Distribution for Lifetime Data. International Journal of Statistical Distributions and Applications. Vol. 4, No. 1, 2018, pp. 6-21. doi: 10.11648/j.ijsd.20180401.12

Received: January 4, 2018; Accepted: February 24, 2018; Published: March 28, 2018

\begin{abstract}
For the first time, the Generalized Gamma Burr III (GGBIII) is introduced as an important model for problems in several areas such as actuarial sciences, meteorology, economics, finance, environmental studies, reliability, and censored data in survival analysis. A review of some existing gamma families have been presented. It was found that the distributions cannot exhibit complicated shapes such as unimodal and modified unimodal shapes which are very common in medical field. The Generalized Gamma Burr III (GGBIII) distribution which includes the family of Zografos and Balakrishnan as special cases is proposed and studied. It is expressed as the linear combination of Burr III distribution and it has a tractable properties. Some mathematical properties of the new distribution including hazard, survival, reverse hazard rate function, moments, moments generating function, mean and median deviations, distribution of the order statistics are presented. Maximum likelihood estimation technique is used to estimate the model parameters and applications to real datasets in order to illustrate the usefulness of the model are presented. Examples and applications as well as comparisons of the GGBIII to the existing Gamma-G families are given.
\end{abstract}

Keywords: Burr III Distribution, Generalized-Gamma Distribution, Censored Data, Maximum Likelihood Estimation

\section{Introduction}

It is known that the Burr III distribution is the third example of solutions of the differential equation defining the Burr system of distribution, [4]. This distribution has been used widely in numerous fields of sciences with different parameterizations using other names. For example, it is used as inverse Burr distribution in the actuarial literature III distribution in low-flow frequency analysis where the lower tail of a distribution is of interest. [13] and kappa distribution in the meteorological literature ([17], 18]). The Burr III distribution has been useful in financial literature, environmental studies, in survival and reliability theory, (such as: $[1,7,10,14,19,26,27]$ ). Recently, [25] proposed the use of the so-called extended Burr.

A system of distributions which contains the Burr XII (BXII) distribution as the most widely used of these distributions was introduced [4]. If a random variable $X$ has the BXII distribution, then $X^{-1}$ has the scaled Burr III (BIII) distribution with cumulative distribution function (cdf) defined (for $x>0$ ) by ([2]).

$$
F(x ; \beta, \delta, \lambda)=\left(1+\left(\frac{x}{\lambda}\right)^{-\delta}\right)^{-\beta}, x>0,
$$

where $\beta>0, \delta>0$ and $\lambda>0$, are shapes and scale parameters respectively. The probability density function corresponding to (1) is given by

$$
f(x ; \beta, \delta, \lambda)=\beta \lambda^{\delta} \delta y^{-\delta-1}\left(1+\left(\frac{x}{\lambda}\right)^{-\delta}\right)^{-\beta-1},
$$

The hazard and reverse hazard functions are given by:

$$
h(x ; \beta, \delta, \lambda)=\frac{\beta \lambda^{\delta} \delta x^{-\delta-1}\left(1+\left(\frac{x}{\lambda}\right)^{-\delta}\right)^{-\beta-1}}{1-\left(1+\left(\frac{x}{\lambda}\right)^{-\delta}\right)^{-\beta}},
$$

and

$$
\tau(x ; \beta, \delta, \lambda)=\beta \lambda^{\delta} \delta y^{-\delta-1}\left(1+\left(\frac{x}{\lambda}\right)^{-\delta}\right)^{-1}
$$

The $r^{t h}$ raw or non-central moments are: 


$$
E\left(Y^{r}\right)=\beta^{*} \lambda^{r} B\left(\beta^{*}+\frac{r}{\delta}, 1-\frac{r}{\delta}\right), r<\delta
$$

Where $B(a, b)=\frac{\Gamma(\mathrm{a}) \Gamma(\mathrm{b})}{\Gamma(a+b)}$.

In this paper, a new class of Burr-type distribution called the Generalized Gamma-Burr III (GGBIII) distribution was presented and applied to some real-life situations.

Motivated by the various applications of Burr III distribution in finance and actuarial sciences, and economics, where distribution plays an important role in size distribution [2]. It is customary to develop models that take into consideration not only shape, and scale but also skewness, kurtosis and tail variations. An obvious reason for generalizing a standard distribution is to provide larger flexibility in modeling real data. It is well known in general that a generalized model is more flexible than the ordinary model and it is preferred by many data analysts in analyzing statistical data, [21]. The gamma distribution is the most effective model for analyzing skewed data [16].

In the last few years, several ways of generating new probability distributions from classic ones were developed and discussed. [12] Studied a distribution family that arises naturally from the distribution of order statistics. The betagenerated family proposed by [5] has been extensively used by many researchers in generalizing distribution. For any baseline continuous distribution $F(x)$ with survival function $1-F(x)$ and density $f(x),[28]$ defined the cumulative distribution function (cdf) and probability density function

$$
g(x)=\frac{1}{\Gamma(\alpha) \theta^{\alpha}}[-\log (1-F(x))]^{\alpha-1}(1-F(x))^{(1 / \theta)-1} f(x),
$$$$
G(x)=\frac{1}{\Gamma(\alpha) \theta^{\alpha}} \int_{0}^{-\log (1-F(x))} z^{\alpha-1} e^{-z / \theta} d z,
$$

respectively, for $\alpha, \theta>0$, where $g(x)=\frac{d G(x)}{d x}, \Gamma(\alpha)=$ $\int_{0}^{\infty} z^{\alpha-1} e^{-z} d z$ denotes the gamma function, and $\gamma(t, \alpha)=$ $\int_{0}^{t} z^{\alpha-1} e^{-z} d z$, denotes the incomplete gamma function. Towards the end, they obtained a natural extension for Dagum distribution, which they called the gamma-Dagum (GD) distribution.

A review of some existing gamma families have been presented, a different type of gamma generalized distribution was introduced [23], a Zografos and Balakrishnan-Dagum (ZB-D) was provided [23] which was modified by [3]. It was found that these distributions cannot exhibit complicated shapes such as unimodal and modified unimodal shapes which are very common in medical field.

In this paper, natural extension of Burr III distribution called the Generalized Gamma Burr III distribution was studied.

This paper is organized as follows. In section 2 , some basic results, the generalized gamma-Burr III (GGBIII) distribution, series expansion and its sub-models, hazard, survival and reverse hazard functions and the quantile function are presented. The moments and moment generating function, mean and median deviations are given in section 3 . Section 4 contains some additional useful results on the distribution of order statistics. In section 5, results on the estimation of the (pdf) as follows

$$
G(x)=\frac{1}{\Gamma(\alpha)} \int_{0}^{-\log (1-F)} t^{\alpha-1} e^{-t} d t, x \in \mathrm{R}, \alpha>0
$$

and

$$
g(x)=\frac{1}{\Gamma(\alpha)}[-\log (1-F(x))]^{\alpha-1} f(x),
$$

respectively. Also, an alternative gamma-generator was proposed [24] and defined the cdf and pdf by

$$
G(x)=1-\frac{1}{\Gamma(\alpha)} \int_{0}^{-\log F(x)} z^{\alpha-1} e^{-z} d z, z \in \mathrm{R}, \alpha>0,
$$

and

$$
g(x)=\frac{1}{\Gamma(\alpha)}[-\log F(x)]^{\alpha-1} f(x),
$$

respectively. The exponentiated exponential (EE) distribution was generalized by [23] and further studied by [9] with cdf $F(x)=\left(1-e^{-\beta x}\right)^{\alpha}$, where $\alpha>0$ and $\beta>0$ inserted into equation (8) to obtain and study the gamma exponentiated exponential (GEE) model. The statistical properties of the gamma - exponentiated Weibull distribution was presented [15].

The distribution with pdf $g(x)$ and $\operatorname{cdf} G(x)$ for any baseline $\operatorname{cdf} F(x)$ define by [3] and $x \in \mathbb{R}$, (for $\alpha>0$ ) as follows

parameters of the GGBIII distribution via the method of maximum likelihood are presented. Applications in different fields are presented in Section 6, followed by discussion in section 7 and conclusion in section 8 .

\section{The Generalized Gamma-G Family}

The standard Gamma distribution of different kind is given by $[8]$

$$
\int_{0}^{\infty} z^{\omega-1} e^{-z^{p} / \theta} d z=\frac{\Gamma(\omega / p) \theta^{\omega / p}}{p}
$$

Then, we write equation (12) as

$$
\frac{1}{\Gamma(\alpha) \theta^{\alpha}} \int_{0}^{x}\left(z^{p}\right)^{\alpha-1} e^{-z^{p} / \theta} d z
$$

This is the known gamma distribution when $p=1$

Equation (13) has the parent distribution with the cdf given as follows

$$
G(x)=\frac{1}{\Gamma(\alpha) \theta^{\alpha}} \int_{0}^{[-\log (1-F(x))]^{p}} z^{\alpha-1} e^{-z / \theta} d z
$$

Equation (14) is the generalization of Gamma-G family of [3] in equation (11) when $p=1$ and also in equation (6) when $p=\theta=1$ [28].

Let $F(x)$ be the cumulative distribution function (CDF) of any random variable $X$. The cdf and pdf of the generalized 
gamma-G family of distributions when $\theta=1$ is given by

$$
\begin{gathered}
G(x)=\frac{1}{\Gamma(\alpha)} \int_{0}^{[-\log (1-F(x))]^{p}} z^{\alpha-1} e^{-z} d z \quad(15) \\
g(x)=\frac{1}{\Gamma(\alpha)}\left[[-\log (1-F(x))]^{p}\right]^{\alpha-1} e^{-[-\log (1-F(x))]^{p}} \frac{d[-\log (1-F(x))]^{p}}{d x} \\
g(x)=\frac{1}{\Gamma(\alpha)}\left[[-\log (1-F(x))]^{p}\right]^{\alpha-1} e^{-[-\log (1-F(x))]^{p}} p[-\log (1-F(x))]^{p-1} \frac{f(x)}{1-F(x)}
\end{gathered}
$$

respectively, $\alpha, \beta, \lambda, \delta, p>0$.

\subsection{Generalized Gamma Burr III Distribution}

The new model is proposed by inserting scaled Burr III distribution into equation (15), the cdf $G_{G G B I I I}(x)$ of the Generalized Gamma-BIII distribution is obtained as follows:

$$
\begin{gathered}
G(x)=\frac{1}{\Gamma(\alpha)} \int_{0}^{\left[-\log \left(1-\left(1+\left(\frac{x}{\lambda}\right)^{-\delta}\right)^{-\beta}\right)\right]^{p}} z^{\alpha-1} e^{-z} d z \\
=\frac{\gamma\left(\left[-\log \left(1-\left(1+\left(\frac{x}{\lambda}\right)^{-\delta}\right)^{-\beta}\right)\right]^{p}, \alpha\right)}{\Gamma(\alpha)}
\end{gathered}
$$

where $\gamma(x, \alpha)=\int_{0}^{x} z^{\alpha-1} e^{-z} d z$ is the incomplete gamma function.

The probability density function is given by

$$
\begin{gathered}
g(x)=\frac{p \beta \delta \lambda^{\delta} x^{-\delta-1}}{\Gamma(\alpha)}\left(1+\left(\frac{x}{\lambda}\right)^{-\delta}\right)^{-\beta-1}\left[\left[-\log \left(1-\left(1+\left(\frac{x}{\lambda}\right)^{-\delta}\right)^{-\beta}\right)\right]^{p}\right]^{\alpha-1} \times \\
e^{-\left[-\log \left(1-\left(1+\left(\frac{x}{\lambda}\right)^{-\delta}\right)^{-\beta}\right)\right]^{p}}\left[1-\left(1+\left(\frac{x}{\lambda}\right)^{-\delta}\right)^{-\beta}\right]^{-1}
\end{gathered}
$$

\section{Shape of GGBIII Distribution}

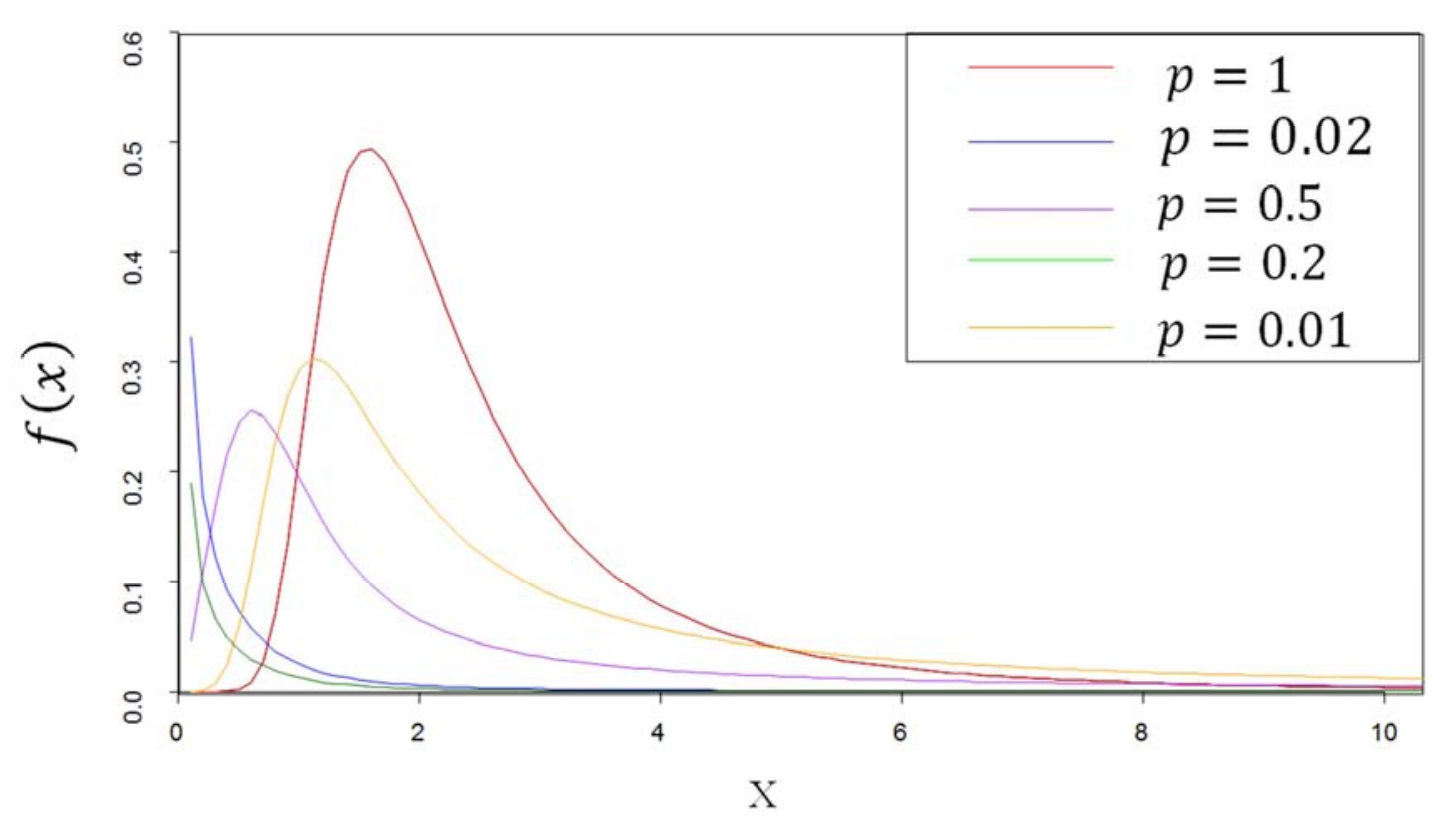

Figure 1. Shape of GGBIII distribution for selected parameters. 
Plots of the density function of the Generalized Gamma Burr III distribution for selected parameters values are given in figure 1. The plot indicates that the GGBIII distribution can be decreasing or right skewed.

\subsection{Expansions of Density Functions}

If a random variable $X$ has the GGBIII density, we write $X \sim G G B I I I(\alpha, \beta, \delta, \lambda, p)$. Let $u=\left(1+\left(\frac{x}{\lambda}\right)^{-\delta}\right)^{-\beta}$, and then using the series representation, it gives

$$
\begin{aligned}
& \log \frac{1}{(1-u)}=\sum_{k=1}^{\infty} \frac{u^{k}}{k}=\sum_{k=0}^{\infty} \frac{u^{k+1}}{k+1} \\
& e^{-[-\log (1-u)]^{p}}=\sum_{h=0}^{\infty}(-1)^{h} \frac{[-\log (1-u)]^{p h}}{h !}, \\
& \frac{1}{1-u}=\sum_{i=0}^{\infty} u^{i} \\
& {[-\log (1-u)]^{p \alpha-1}=u^{p \alpha-1}\left[\sum_{j=0}^{\infty}\left(\begin{array}{c}
p \alpha-1 \\
j
\end{array}\right) u^{j}\left(\sum_{s=0}^{\infty} \frac{u^{s}}{s+2}\right)^{j}\right]}
\end{aligned}
$$

and applying the result on the power series raised to a positive integer, with $c_{s}=(s+2)^{-1}$, that is

$$
\left(\sum_{s=0}^{\infty} c_{s} u^{s}\right)^{j}=\sum_{s=0}^{\infty} a_{s, j} u^{s}
$$

where $a_{s, j}=\left(s c_{0}\right)^{-1} \sum_{l=1}^{s}[j(l+1)-s] c_{l} a_{s-l, j}$, and $a_{0, j}=c_{0}^{j}$, [8], the GGBIII pdf can be written as

$$
\begin{gathered}
g_{G G B I I I}(x)=\frac{p \lambda^{\delta} \beta \delta x^{-\delta-1}\left[1+\left(\frac{x}{\lambda}\right)^{-\delta}\right]^{-\beta-1}}{\Gamma(\alpha)} u^{p \alpha-1} \times \sum_{j=0}^{\infty} \sum_{s=0}^{\infty}\left(\begin{array}{c}
p \alpha-1 \\
j
\end{array}\right) a_{s, j} u^{j+s} \sum_{h=0}^{\infty}(-1)^{h} \frac{[-\log (1-u)]^{p h}}{h !} \sum_{i=0}^{\infty} u^{i} \\
=\frac{p \lambda^{\delta} \beta \delta x^{-\delta-1}\left[1+\left(\frac{x}{\lambda}\right)^{-\delta}\right]^{-\beta-1}}{\Gamma(\alpha)} \times \sum_{j=0}^{\infty} \sum_{s,=0}^{\infty} \sum_{h=0}^{\infty} \sum_{i=0}^{\infty}\left(\begin{array}{c}
p \alpha-1 \\
j
\end{array}\right)(-1)^{h} \frac{[-\log (1-u)]^{p h}}{h !} a_{s, j} u^{p \alpha+s+j+i-1} \\
=\sum_{j=0}^{\infty} \sum_{s=0}^{\infty} \sum_{h=0}^{\infty} \sum_{i=0}^{\infty}(-1)^{h}\left(\begin{array}{c}
p \alpha-1 \\
j
\end{array}\right) \frac{p \lambda^{\delta} \beta \delta x^{-\delta-1}\left[1+\left(\frac{x}{\lambda}\right)^{-\delta}\right]^{-\beta-1}}{\Gamma(\alpha)} \times \frac{[-\log (1-u)]^{p h}}{h !} a_{s, j} u^{p \alpha+s+j+i-1} \\
=\sum_{j=0}^{\infty} \sum_{s=0}^{\infty} \sum_{h=0}^{\infty} \sum_{i=0}^{\infty}(-1)^{h}\left(\begin{array}{c}
p \alpha-1 \\
j
\end{array}\right) \frac{p \lambda^{\delta} \beta \delta x^{-\delta-1}\left[1+\left(\frac{x}{\lambda}\right)^{-\delta}\right]^{-\beta-1}}{\Gamma(\alpha)} \times \frac{[-\log (1-u)]^{p h}}{h !} a_{s, j}\left[1+\left(\frac{x}{\lambda}\right)^{-\delta}\right]^{-\beta p \alpha+\beta s+\beta j+\beta i+\beta} \\
=\sum_{j=0}^{\infty} \sum_{s=0}^{\infty} \sum_{h=0}^{\infty} \sum_{i=0}^{\infty}(-1)^{h}\left(\begin{array}{c}
p \alpha-1 \\
j
\end{array}\right) \frac{p \lambda^{\delta} \beta \delta x^{-\delta-1}}{\Gamma(\alpha)} \times a_{s, j}\left[1+\left(\frac{x}{\lambda}\right)^{-\delta}\right]^{-\beta(p \alpha+s+j+i)-1} \frac{[-\log (1-u)]^{p h}}{h !} \\
=\sum_{j=0}^{\infty} \sum_{s=0}^{\infty} \sum_{h=0}^{\infty} \sum_{i=0}^{\infty}(-1)^{h}\left(\begin{array}{c}
p \alpha-1 \\
j
\end{array}\right) \frac{p \lambda^{\delta} \beta(p \alpha+s+j+i) \delta x^{-\delta-1}}{\Gamma(\alpha)(p \alpha+s+j+i)} a_{s, j} \times\left[1+\left(\frac{x}{\lambda}\right)^{-\delta}\right]^{-\beta(p \alpha+s+j+i)-1} \frac{[-\log (1-u)]^{p h}}{h !} \\
=\sum_{j=0}^{\infty} \sum_{s=0}^{\infty} \sum_{h=0}^{\infty} \sum_{i=0}^{\infty} \frac{(-1)^{h}}{h !}\left(\begin{array}{c}
p \alpha-1 \\
j
\end{array}\right) \frac{p \lambda^{\delta} \beta(p \alpha+s+j+i) \delta x^{-\delta-1}}{\Gamma(\alpha)(p \alpha+s+j+i)} a_{s, j} \times\left[1+\left(\frac{x}{\lambda}\right)^{-\delta}\right]^{-\beta(p \alpha+s+j+i)-1}\left[-\log \left(1-\left(1+\left(\frac{x}{\lambda}\right)^{-\delta}\right)\right.\right.
\end{gathered}
$$

where $f(x ; \lambda, \beta(p \alpha+j+s+i), \delta)$ is the Burr III pdf with parameters $\lambda, \beta(p \alpha+j+s+i)$ and $\delta$. Let $C=\left\{(j, h, s, i) \epsilon Z_{+}^{3}\right\}$

$$
\varphi_{v}=\frac{(-1)^{h}}{h !}\left(\begin{array}{c}
p \alpha-1 \\
j
\end{array}\right) \frac{a_{s, j}}{\Gamma(\alpha)(p \alpha+s+j+i)^{\prime}}
$$

and 


$$
g_{G G B I I I}(x)=\sum_{v \in C} \varphi_{v} f(x ; \lambda, \beta(p \alpha+j+s+i), \delta) .
$$

\subsection{Hazard and Reverse Hazard Functions}

Let $X$ be a continuous random variable with distribution function $F$, and probability density function (pdf) $f$, then the hazard function, reverse hazard function are given by $h_{F}(x)=f(x) /(1-F(x))$. The hazard and reverse hazard function of the GGBIII distribution are

$$
h(x)=\frac{\frac{p \beta \lambda^{\delta} \delta x^{-\delta-1}\left(1+\left(\frac{x}{\lambda}\right)^{-\delta}\right)^{-\beta-1}}{\left(1-\left(1+\left(\frac{x}{\lambda}\right)^{-\delta}\right)^{-\beta}\right)} \times\left[-\log \left(1-\left(1+\left(\frac{x}{\lambda}\right)^{-\delta}\right)^{-\beta}\right)\right]^{p \alpha-1} \times e^{-\left[-\log \left(1-\left(1+\left(\frac{x}{\lambda}\right)^{-\delta}\right)^{-\beta}\right)\right]^{p}}}{\Gamma(\alpha)-\gamma\left(\left[-\log \left[1-\left(1+\left(\frac{x}{\lambda}\right)^{-\delta}\right)^{-\beta}\right]\right]^{p}, \alpha\right)}
$$

and

$$
\tau(x)=\frac{\frac{p \beta \lambda^{\delta} \delta x^{-\delta-1}\left(1+\left(\frac{x}{\lambda}\right)^{-\delta}\right)^{-\beta-1}}{\left(1-\left(1+\left(\frac{x}{\lambda}\right)^{-\delta}\right)^{-\beta}\right)} \times\left[-\log \left(1-\left(1+\left(\frac{x}{\lambda}\right)^{-\delta}\right)^{-\beta}\right)\right]^{p \alpha-1} \times e^{-\left[-\log \left(1-\left(1+\left(\frac{x}{\lambda}\right)^{-\delta}\right)^{-\beta}\right)\right]^{p}}}{\gamma\left(\left[-\log \left[1-\left(1+\left(\frac{x}{\lambda}\right)^{-\delta}\right)^{-\beta}\right]\right]^{p}, \alpha\right)}
$$

respectively, for $x \geq 0, \beta \geq 0, \delta \geq 0, \alpha \geq 0, p \geq 0$, and $\lambda \geq 0$.

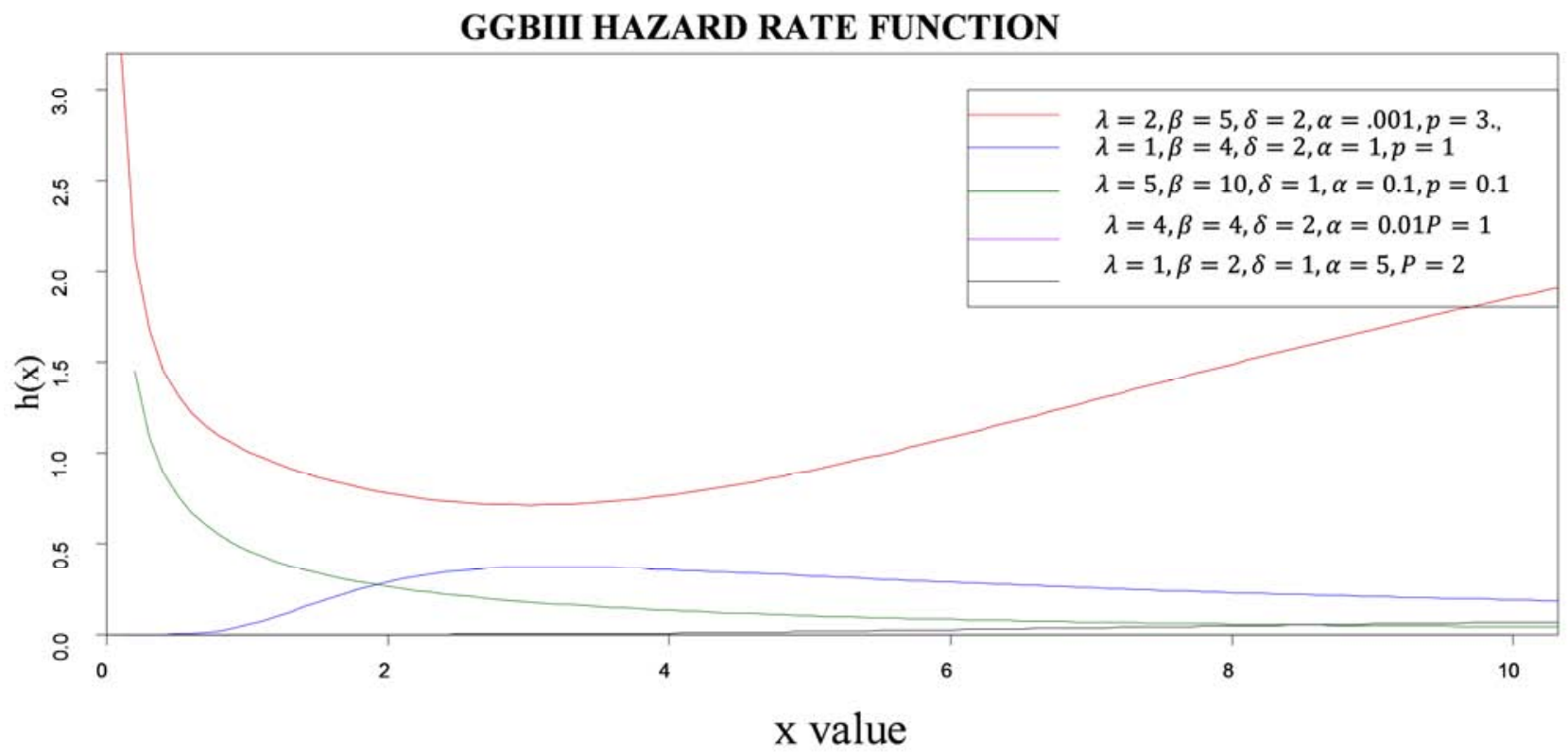

Figure 2. THE Plot of Hazard Rate Function of GGBIII.

The plots show various shapes including monotonically decreasing, monotonically increasing, and bathtub followed by upside down bathtub shapes for five combinations of values of the parameters. This very attractive flexibility makes the GGBIII hazard rate function useful and suitable for monotonic and non-monotone empirical hazard behaviors which are more likely to be encountered or observed in real life situations.

\subsection{GGBIII Quantile Function}

Let $X$ be a random variable with distribution function $F$, and let $q \in(0,1)$. A value of $x$ such that $F\left(x^{-}\right)=$ $P(x<x) \leq q$ and $F(x)=P(X \leq x) \geq q$ is called a quantile of order $q$ for the distribution. Roughly speaking, a quantile of order $q$ is a value where the graph of the cumulative distribution function crosses (or jumps over) $q$.

The quantile function of GGBIII distribution is obtained by solving the equation

Thus, the quantile function is given by 


$$
\begin{aligned}
& F^{-1}(q)=\min \{x \in R: F(x) \geq q\}, q \in(0,1) \\
& Q_{G G B I I I}\left(x_{p}\right)=q, 0<q<1 . \\
& Q_{G G B I I I}(q)=\lambda\left[\left(1-e^{-u^{1 / p}}\right)^{-1 / \beta}-1\right]^{-1 / \delta}
\end{aligned}
$$

then $\gamma(u, \alpha)=q \Gamma(\alpha)$, where $u=\gamma^{-1}(q \Gamma(\alpha), \alpha)$.

\section{Moments, Moment Generating Function, Mean and Median Deviations}

In this section, the moments, moment generating function, mean and median deviations for the GGBIII distribution were presented.

\subsection{Moments and Moment Generating Function}

As with any other distribution, many of the interesting characteristics and features of the GGBIII distribution can be studied through the moments. Let $\beta^{*}=\beta(p \alpha+j+s+i)$, and $Y \sim B \operatorname{BII}\left(\delta, \lambda, \beta^{*}\right)$. Then the $r^{\text {th }}$ moment of the random variable $Y$ is

$$
E\left(Y^{r}\right)=\beta^{*} \lambda^{r} B\left(\beta^{*}+\frac{r}{\delta}, 1-\frac{r}{\delta}\right), r<\delta
$$

So that the $r^{\text {th }}$ raw moment is thus given by the following

$$
E\left(X^{r}\right)=\sum_{v \in C} \varphi_{v} \beta^{*} \lambda^{r} B\left(\beta^{*}+\frac{r}{\delta}, 1-\frac{r}{\delta}\right) r<\delta,
$$

The moment generating function of the BGBIII distribution is given by

$$
\begin{aligned}
M_{X}(t)=E\left(e^{t X}\right)= & E\left[1+t X+\frac{(t X)^{2}}{2 !}+\frac{(t X)^{3}}{3 !}+\cdots\right], \\
& =\sum_{r=1}^{\infty} \frac{t^{r}}{r !} E\left(X^{r}\right),
\end{aligned}
$$

then we have,

$$
\sum_{r=0}^{\infty} \sum_{v \in C} \frac{t^{r}}{r !} \varphi_{v} \beta^{*} \lambda^{r} B\left(\beta^{*}+r / \delta, 1-r / \delta\right) r<\delta,
$$

\subsection{Mean and Median Deviations}

\subsubsection{Mean Deviation}

If $\mathrm{X}$ has the GGBIII distribution, we derive the mean deviation about the mean $\mu$ by

$$
\delta_{1}=\int_{0}^{\infty}|x-\mu| g_{G D}(x) d x=2 \mu G_{G G B I I I}(\mu)-2 \mu+2 T(\mu),
$$

Where $\mu=E(X)$ and $T(\mu)=\int_{\mu}^{\infty} x \cdot g_{G G B I I I}(x) d x$. Let $\beta^{*}=\beta(p \alpha+j+s+i)$, then

$$
\begin{gathered}
T(\mu)=\sum_{v \in C} \varphi_{v} T_{G G B I I I\left(\beta^{*}, \delta, \lambda\right)}(\mu) \\
=\sum_{v \in C} \varphi_{v} \beta^{*} \lambda\left[B\left(\beta^{*}+1 / \delta, 1-1 / \delta\right)-B\left(t(\mu) ; \beta^{*}+1 / \delta, 1-1 / \delta\right)\right] \\
\delta_{1}=2 \sum_{v \in C} \varphi_{v} \beta^{*} \lambda\left[B\left(\beta^{*}+1 / \delta, 1-1 / \delta\right)\right]\left[\frac{\gamma\left(\left[-\log \left[1-\left(1+\left(\frac{\mu}{\lambda}\right)^{-\delta}\right)^{-\beta}\right]\right]^{p}, \alpha\right)}{\Gamma(\alpha)}-1\right] \\
+2 \sum_{v \in C} \varphi_{v} \beta^{*} \lambda\left[B\left(\beta^{*}+1 / \delta, 1-1 / \delta\right)-B\left(t(\mu) ; \beta^{*}+1 / \delta, 1-1 / \delta\right)\right]
\end{gathered}
$$

Where $t(\mu)=\left(1+\left(\frac{\mu}{\lambda}\right)^{-\delta}\right)^{-1}$ and $B(x ; a, b)=\int_{0}^{x} t^{a-1}(1-t)^{b-1} d t$.

\subsubsection{Median Deviation}

If $X$ has the GGBIII distribution, then the median deviation about the median $M$ is derived by

$$
\delta_{2}=\int_{0}^{\infty}|x-M| g_{G D}(x) d x=2 T(M)-\mu,
$$

where $T(M)=\int_{M}^{\infty} x \cdot g_{G G B I I I}(x) d x$

$$
\begin{gathered}
M=Q_{G G B I I I}(0.5)=\lambda\left[\left(1-e^{-\left[\gamma^{-1}(0.5 \Gamma(\alpha), \alpha)\right]^{1 / p}}\right)^{-1 / \beta}-1\right]^{-1 / \delta} \\
T(M)=\sum_{v \in C} \varphi_{v} T_{B I I I\left(\beta^{*}, \delta, \lambda\right)}(M) \\
=\sum_{v \in C} \varphi_{v} \beta^{*} \lambda\left[B\left(\beta^{*}+1 / \delta, 1-1 / \delta\right)-B\left(t(M) ; \beta^{*}+1 / \delta, 1-1 / \delta\right)\right]
\end{gathered}
$$


$2 \sum_{v \in C} \varphi_{v} \beta^{*} \lambda\left[B\left(\beta^{*}+1 / \delta, 1-1 / \delta\right)-B\left(t(M) ; \beta^{*}+1 / \delta, 1-1 / \delta\right)\right]-\sum_{v \in C} \varphi_{v} \beta^{*} \lambda\left[B\left(\beta^{*}+1 / \delta, 1-1 / \delta\right)\right]$

\section{Order Statistics of Ggbiii Distribution}

Definition: Let $\left(\mathrm{y}_{1}, \ldots, \mathrm{y}_{\mathrm{n}}\right)$ be a random sample from the GGBIII distribution with pdf $\mathrm{f}(\mathrm{y})$ defined over the interval $-\infty$ to $\infty$. A rearrangement of the random sample into $\left(x_{1}, \ldots, x_{n}\right)$ i.e. $-\infty<x_{1}, x_{2}, \ldots x_{n}<\infty$ is known as the ordered transformation of the random sample and $\mathrm{x}_{1}, \mathrm{x}_{2}, \ldots, \mathrm{x}_{\mathrm{n}}$ are called ordered statistics.

Theorem: Let $x_{1}, x_{2}, \ldots, x_{n}$ be ordered statistics of a random sample $\left(\mathrm{y}_{1}, \ldots, \mathrm{y}_{\mathrm{n}}\right)$ from a GGBIII distribution with pdf $g(x)$, the joint pdf is given as

$$
f\left(x_{1}, x_{2}, \ldots, x_{n}\right)=n ! g\left(x_{1}\right) g\left(x_{2}\right) \ldots g\left(x_{n}\right) \text { for }-\infty<x_{1}<x_{2}<\cdots<x_{n}<\infty .
$$

The general formula for order statistics is given by

$$
f_{i ; n}(x)=\frac{n ! g(x)}{(n-i) !(i-1) !}[G(x)]^{i-1}[1-G(x)]^{n-i},
$$

Again, using the binomial expansion to the second factor, it gives

$$
\begin{gathered}
f_{i ; n}(x)=\frac{n ! g(x)}{(n-i) !(i-1) !} \sum_{j=0}^{n-i}(-1)^{j}\left(\begin{array}{c}
n-i \\
j
\end{array}\right)[G(x)]^{i+j-1}, \\
f_{i ; n}(x)=\frac{n ! g(x)}{(n-i) !(i-1) !} \sum_{j=0}^{n-i}(-1)^{j}\left(\begin{array}{c}
n-i \\
j
\end{array}\right)\left[\frac{\gamma\left([-\log (1-F(x))]^{p}, \alpha\right.}{\Gamma(\alpha)}\right]^{i+j-1}
\end{gathered}
$$

From [8]

$$
\gamma(x, \alpha)=\sum_{k=o}^{\infty} \frac{(-1)^{k} x^{k+\alpha}}{(k+\alpha) k !}
$$

and if $d_{k}=(-1)^{k} /((k+\alpha) k !)$, then

$$
\begin{gathered}
f_{i ; n}(x)=\frac{n ! g(x)}{(n-i) !(i-1) !} \sum_{j=0}^{n-i}\left(\begin{array}{c}
n-i \\
j
\end{array}\right) \frac{(-1) j^{j}}{[\Gamma(\alpha)]^{i+j-1}}\left([[-\log (1-F(x))]]^{p}\right)^{\alpha(i+j-1)} \times\left[\sum_{k=0}^{\infty} \frac{(-1)^{k}\left(\left[[-\log (1-F(x))]^{p}\right]\right)^{k}}{(k+\alpha) k !}\right]^{i+j-1} \\
f_{i ; n}(x)=\frac{n ! g(x)}{(n-i) !(i-1) !} \sum_{j=0}^{n-i}\left(\begin{array}{c}
n-i \\
j
\end{array}\right) \frac{(-1)^{j}}{[\Gamma(\alpha)]^{i+j-1}}\left([-\log (1-F(x))]^{p}\right)^{\alpha(i+j-1)} \times \sum_{k=0}^{\infty}\left([-\log (1-F(x))]^{p}\right)^{k}, \\
f_{i ; n}(x)=\frac{n ! g(x)}{(n-i) !(i-1) !} \sum_{j=0}^{n-i} \sum_{k=0}^{\infty}\left(\begin{array}{c}
n-i \\
j
\end{array}\right) \frac{(-1)^{j} d_{k, n-i+j}}{[\Gamma(\alpha)]^{i+j-1}}\left([-\log (1-F(x))]^{p}\right)^{\alpha(i+j-1)+k} \\
=\frac{n ! p([-\log (1-F(x))])^{p \alpha-1} e^{-[-\log (1-F(x))]^{p}}[1-F(x)]^{-1} f(x)}{(n-i) !(i-1) ! \Gamma(\alpha)} \sum_{j=0}^{n-i} \sum_{k=0}^{\infty}\left(\begin{array}{c}
n-i \\
j
\end{array}\right) \frac{(-1)^{j} d_{m i, i+1}}{[\Gamma(\alpha)]^{i+j-1}} \times\left([-\log (1-F(x))]^{p}\right)^{\alpha(i+j-1)+k} \\
=\frac{n !}{(n-i) !(i-1) !} \sum_{j=0}^{n-i} \sum_{k=0}^{\infty}\left(\begin{array}{c}
n-i \\
j
\end{array}\right) \frac{(-1)^{j} d_{m, i+j-1}}{[\Gamma(\alpha)]^{i+j}}\left([-\log (1-F(x))]^{p}\right)^{p \alpha(i+j)+k-1} e^{-[-\log (1-F(x))]^{p}[1-F(x)]^{-1} f(x)} \\
=\frac{n !}{(n-i) !(i-1) !} \sum_{j=0}^{n-i} \sum_{k=0}^{\infty}\left(\begin{array}{c}
n-i \\
j
\end{array}\right) \frac{(-1)^{j} d_{m i, i+j-1}}{[\Gamma(\alpha)]^{i+j}} \times \frac{\Gamma((\alpha(i+j)+k))}{\Gamma((\alpha(i+j)+k))}[-\log (1-F(x))]^{p \alpha(i+j-1)+k-1} \times e^{-[-\log (1-F(x))]^{p} \times[1-F(x)]^{-1} f(x)}
\end{gathered}
$$

That is,

$$
f_{i, n}(x)=\frac{n !}{(n-i) !(i-1) !} \sum_{j=0}^{n-i} \sum_{k=0}^{\infty}\left(\begin{array}{c}
n-i \\
j
\end{array}\right) \frac{(-1)^{j} d_{m, i+j-1} \Gamma(\alpha(i+j)+k)}{[\Gamma(\alpha)]^{i+j}} \times f(x ; \alpha(i+j)+k, p, \beta, \lambda, \delta),
$$

where $f(x ; \alpha(i+j)+k, p, \beta, \lambda, \delta)$ is the GGBIII pdf with parameters $\beta, \delta, \lambda, p$ and shape parameter $\alpha^{*}=\alpha(i+j)+k$.

$$
=\frac{n !}{(n-i) !(i-1) !} \sum_{j=0}^{n-i} \sum_{k=0}^{\infty}\left(\begin{array}{c}
n-i \\
j
\end{array}\right) \frac{(-1)^{j} d_{m, i+j-1} \Gamma((\alpha(i+j)+k))}{[\Gamma(\alpha)]^{i+j}} \frac{p \beta \delta \lambda^{\delta} x^{-\delta-1}\left(1+\left(\frac{x}{\lambda}\right)^{-\delta}\right)^{-\beta-1}}{\Gamma((\alpha(i+j)+k))}
$$




$$
\left.\left[-\log \left(1-\left(1+\left(\frac{x}{\lambda}\right)^{-\delta}\right)^{-\beta}\right)\right]^{p \alpha(i+j-1)+k-1} e^{-\left[-\log \left(1-\left(1+\left(\frac{x}{\lambda}\right)^{-\delta}\right)^{-\beta}\right)\right.}\right]^{p}\left[1-\left(1+\left(\frac{x}{\lambda}\right)^{-\delta}\right)^{-\beta}\right]^{-1}
$$

\section{Maximum Likelihood Estimation}

The maximum likelihood estimation (MLE) is one of the most widely used estimation method for finding the unknown parameters. Consider a random sample $x_{1}, x_{2}, \ldots, x_{n}$ from the generalized gamma-Dagum distribution.

The likelihood function is given by

$$
\begin{gathered}
L(\beta, \alpha, \delta, \lambda, p)=\frac{\left(p \beta \lambda^{\delta} \delta\right)^{n}}{[\Gamma(\alpha)]^{n}} \\
\prod_{i=1}^{n}\left\{x_{i}^{-\delta-1}\left[1+\left(\frac{x_{i}}{\lambda}\right)^{-\delta}\right]^{-\beta-1}\left[-\log \left(1-\left(1+\left(\frac{x_{i}}{\lambda}\right)^{-\delta}\right)^{-\beta}\right)\right]^{p \alpha-1}\left[1-\left(1+\lambda x_{i}^{-\delta}\right)^{-\beta}\right]^{-1} \times e^{-\left[-\log \left(1-\left(1+\left(\frac{x_{i}}{\lambda}\right)^{-\delta}\right)^{-\beta}\right)\right]^{p}}\right\}
\end{gathered}
$$

where $\Theta=(p, \alpha, \beta, \delta, \lambda)^{T}$

Now, the log-likelihood function denoted by $\ell$

$$
\ell=\log [L(\Theta)]
$$

$$
\begin{aligned}
& L(\theta)=n \log \left(\lambda^{\delta}\right)+n \log (p)+n \log (\beta)+n \log (\delta)-n \log \Gamma(\alpha)-(\delta+1) \sum_{i=1}^{n} \log \left(x_{i}\right)-(\beta+1) \sum_{i=1}^{n} \log \left(1+\left(\frac{x_{i}}{\lambda}\right)^{-\delta}\right)+ \\
& (p \alpha-1) \sum_{i=1}^{n} \log \left[-\log \left(1-\left(1+\lambda x_{i}^{-\delta}\right)^{-\beta}\right)\right]-\sum_{i=0}^{n} \log \left[1-\left(1+\lambda x_{i}^{-\delta}\right)^{-\beta}\right]-\sum_{i=1}^{n}\left[-\log \left(1-\left(1+\lambda x_{i}^{-\delta}\right)^{-\beta}\right)\right]^{p}(57)
\end{aligned}
$$

The partial derivatives of $\ell$ with respect to the parameters are

$$
\begin{aligned}
& \frac{\partial l}{\partial \alpha}=-n \frac{\Gamma^{\prime}(\alpha)}{\Gamma(\alpha)}+p \sum_{i=0}^{n} \log \left[-\log \left(1-\left(1+\left(\frac{x_{i}}{\lambda}\right)^{-\delta}\right)^{-\beta}\right)\right] \\
& \frac{\partial l}{\partial \beta}=\frac{n}{\beta}-\sum_{i=1}^{n} \log \left(1+\left(\frac{x_{i}}{\lambda}\right)^{-\delta}\right)-(p \alpha-1) \sum_{i=0}^{n} \frac{\left(1+\left(\frac{x_{i}}{\lambda}\right)^{-\delta}\right)^{-\beta} \log \left(1+\left(\frac{x_{i}}{\lambda}\right)^{-\delta}\right)}{\left(1-\left(1+\left(\frac{x_{i}}{\lambda}\right)^{-\delta}\right)^{-\beta}\right) \log \left(1-\left(1+\left(\frac{x_{i}}{\lambda}\right)^{-\delta}\right)^{-\beta}\right)}- \\
& \sum_{i=0}^{n} \frac{\left(1+\left(\frac{x_{i}}{\lambda}\right)^{-\delta}\right)^{-\beta} \log \left(1+\left(\frac{x_{i}}{\lambda}\right)^{-\delta}\right)}{\left(1-\left(1+\left(\frac{x_{i}}{\lambda}\right)^{-\delta}\right)^{-\beta}\right)}-p \sum_{i=1}^{n} \frac{\left[-\log \left(1-\left(1+\left(\frac{x_{i}}{\lambda}\right)^{-\delta}\right)^{-\beta}\right)\right]^{p-1}\left(1+\left(\frac{x_{i}}{\lambda}\right)^{-\delta}\right)^{-\beta} \log \left(1+\left(\frac{x_{i}}{\lambda}\right)^{-\delta}\right)}{\left(1-\left(1+\left(\frac{x_{i}}{\lambda}\right)^{-\delta}\right)^{-\beta}\right)} \\
& \frac{\partial l}{\partial \delta}=\frac{n}{\delta}-n \log \lambda-\sum_{i=1}^{n} \log x_{i}+(\beta+1) \sum_{i=1}^{n} \frac{\lambda x_{i}^{-\delta} \log x_{i}}{1+\left(\frac{x^{i}}{\lambda}\right)^{-\delta}}+(p \alpha-1) \sum_{i=1}^{n} \frac{\left(1+\left(\frac{x_{i}}{\lambda}\right)^{-\delta}\right)^{-\beta}\left(\frac{x_{i}}{\lambda}\right)^{-\delta} \log \left(\frac{x_{i}}{\lambda}\right)}{\left[1-\left(1+\left(\frac{x_{i}}{\lambda}\right)^{-\delta}\right)^{-\beta}\right]\left(\log \left(1-\left(1+\left(\frac{x_{i}}{\lambda}\right)^{-\delta}\right)^{-\beta}\right)\right)} \\
& -p \sum_{i=1}^{n} \frac{\left[-\log \left(1-\left(1+\left(\frac{x_{i}}{\lambda}\right)^{-\delta}\right)^{-\beta}\right)\right]^{p-1}\left(\frac{x_{i}}{\lambda}\right)^{-\delta}\left(1+\left(\frac{x_{i}}{\lambda}\right)^{-\delta}\right)^{-\beta} \log \left(\frac{x_{i}}{\lambda}\right)}{\left[1-\left(1+\left(\frac{x_{i}}{\lambda}\right)^{-\delta}\right)^{-\beta}\right]}+\sum_{i=1}^{n} \frac{\left(1+\left(\frac{x_{i}}{\lambda}\right)^{-\delta}\right)^{-\beta}\left(\frac{x_{i}}{\lambda}\right)^{-\delta} \log \left(\frac{x_{i}}{\lambda}\right)}{\left(1-\left(1+\left(\frac{x_{i}}{\lambda}\right)^{-\delta}\right)^{-\beta}\right)} \\
& \left.\left.\frac{\partial l}{\partial \lambda}=\frac{n \delta}{\lambda}-(\beta+1) \sum_{i=1}^{n} \frac{\left(\frac{x_{i}}{\lambda}\right)^{-\delta} \frac{\delta}{\lambda}}{1+\left(\frac{x_{i}}{\lambda}\right)^{-\delta}}+(p \alpha-1) \sum_{i=1}^{n} \frac{\beta \frac{\delta}{\lambda}\left(\frac{x_{i}}{\lambda}\right)^{-\delta}\left(1+\lambda x_{i}^{-\delta}\right)^{-\beta}}{\left(1-\left(1+\left(\frac{x_{i}}{\lambda}\right)^{-\delta}\right)^{-\beta}\right)\left[-\log \left(1-\left(1+\left(\frac{x_{i}}{\lambda}\right)^{-\delta}\right)\right.\right.}\right)\right]
\end{aligned}
$$




$$
p \sum_{i=1}^{n} \frac{\left[-\log \left(1-\left(1+\left(\frac{x_{i}}{\lambda}\right)^{-\delta}\right)^{-\beta}\right)\right]^{p-1}\left(1+\left(\frac{x_{i}}{\lambda}\right)^{-\delta}\right)^{-\beta}\left(\frac{x_{i}}{\lambda}\right)^{-\delta} \frac{\delta}{\lambda}}{\left[1-\left(1+\left(\frac{x_{i}}{\lambda}\right)^{-\delta}\right)^{-\beta}\right]}-\sum_{i=1}^{n} \frac{\left(1+\lambda x_{i}^{-\delta}\right)^{-\beta}\left(\frac{x_{i}}{\lambda}\right)^{-\delta_{\delta}} \frac{\delta}{\lambda}}{\left[1-\left(1+\left(\frac{x_{i}}{\lambda}\right)^{-\delta}\right)^{-\beta}\right]}
$$

$\frac{\partial l}{\partial p}=\frac{n}{p}+\alpha \sum_{i=0}^{n} \log \left[-\log \left[1-\left(1+\left(\frac{x_{i}}{\lambda}\right)^{-\delta}\right)^{-\beta}\right]\right]+\sum_{i=1}^{n}\left(\log \left[1-\left(1+\left(\frac{x_{i}}{\lambda}\right)^{-\delta}\right)^{-\beta}\right]\right)^{p} \log \left(\log \left[1-\left(1+\left(\frac{x_{i}}{\lambda}\right)^{-\delta}\right)^{-\beta}\right]\right)$

respectively. The MLE of the parameters $\beta, \delta, \lambda, p, \alpha$ will be obtained and they are denoted by $\hat{\beta}, \hat{\lambda}, \hat{p}, \hat{\delta}, \hat{\alpha}$.

Asymptotic confidence intervals

The asymptotic confidence intervals for the parameters of the GGBIII distribution are presented. The expectations in the Fisher Information Matrix (FIM) will now be obtained numerically. The approximate $100(1-\eta) \%$ two-sided confidence intervals for $\beta, \delta, \lambda, \alpha, p$. are given by:

$$
\hat{\beta} \pm Z_{\frac{n}{2}} \sqrt{I_{\beta \beta}^{-1}(\widehat{\Theta})}, \hat{\lambda} \pm Z_{\frac{n}{2}} \sqrt{I_{\lambda \lambda}^{-1}(\widehat{\Theta})}, \hat{\delta} \pm Z_{\frac{n}{2}} \sqrt{I_{\delta \delta}^{-1}(\widehat{\Theta})}, \hat{\alpha} \pm Z_{\frac{n}{2}} \sqrt{I_{\alpha \alpha}^{-1}(\widehat{\Theta})}, \hat{p} \pm Z_{\frac{n}{2}} \sqrt{I_{p p}^{-1}(\widehat{\Theta})}
$$

Under the usual regularity conditions, the well-known asymptotic properties of the maximum likelihood method ensure that $\sqrt{n}\left(\widehat{\Theta_{n}}-\Theta\right) \stackrel{d}{\rightarrow} N\left(0, \Sigma_{\Theta}\right)$, where the $\Sigma_{\Theta}=$ $[I(\Theta)]^{-1}$ is the asymptotic variance-covariance matrix and

$$
I(\Theta)=\left(\begin{array}{cccc}
J_{\alpha \alpha} & J_{\beta \alpha} & J_{\delta \alpha} J_{\lambda \alpha} & J_{p \alpha} \\
J_{\alpha \beta} & J_{\beta \beta} & J_{\delta \beta} J_{\lambda \beta} & J_{p \beta} \\
J_{\alpha \delta} & J_{\beta \delta} & J_{\delta \delta} J_{\lambda \delta} & J_{p \delta} \\
J_{\alpha \lambda} & J_{\beta \lambda} & J_{\delta \lambda} J_{\lambda \lambda} & J_{p \lambda} \\
J_{\alpha p} & J_{\beta p} & J_{\delta p} J_{\lambda p} & J_{p p}
\end{array}\right)
$$

We can use the likelihood ratio (LR) test to compare the fit of the GD distribution with its sub-models for a given data set. The LR test rejects the null hypothesis if $\omega>\chi_{c}^{2}$ where $\chi_{c}^{2}$ denote the upper $100 \%$ point of the $\chi^{2}$ distribution with 2 degrees of freedom.

\section{Applications}

In this section, we present applications of the proposed
GGBIII distribution and compare it to the existing Gamma-G families in real data sets to illustrate its potentiality and robustness. The maximum likelihood estimates (MLEs) of the GGBIII parameters $\alpha, \beta, \delta, \lambda$ and $p$ are computed by maximizing the objective function via the sub-routine NLMIXED in SAS. The estimated values of the parameters (standard error in parenthesis), -2log-likelihood statistic, Akaike Information Criterion, Bayesian Information Criterion, and Corrected Akaike Information Criterion are presented. Also, presented are values of Likelihood Ratio test, Kolmogorov-Smirnov, Cramer-Von Mises, AndersonDarling statistic for hypothesis test which were obtained using a package fitdistrplus in R. In order to compare the models above with the proposed model, we applied formal goodness-of-fit tests to verify which distribution fits better to the real data sets. Here, we consider the Anderson-Darling $(A)$, Cram é r-von Mises $(W)$ and Kolmogorov-Smirnov statistics $K S$. In general, the distribution which has the

\begin{tabular}{|c|c|c|c|c|c|c|c|c|c|c|}
\hline Data & $\mathbf{N}$ & Mean & Median & Mode & SD & Var & Skewness & Kurtosis & Min & $\operatorname{Max}$ \\
\hline Myelo & 33 & 40.88 & 22.00 & 4.00 & 46.70 & 2181.17 & 1.22 & 0.35 & 1 & 156 \\
\hline Aircon & 188 & 92.07 & 54.00 & 14.00 & 107.92 & 11645.93 & 2.16 & 5.19 & 1 & 603 \\
\hline Airc & 30 & 85.93 & 22.00 & 11.00 & 165.72 & 27463.58 & 4.06 & 18.83 & 1 & 877 \\
\hline Comp & 50 & 3.34 & 1.41 & & 4.181 & 17.48 & 1.46 & 1.33 & 0.04 & 15.04 \\
\hline Cancer & 1207 & 46.96 & 42.97 & 18.67 & 29.63 & 878.46 & 0.63 & -0.24 & 2.63 & 133.80 \\
\hline
\end{tabular}
smallest values of these statistics is the better fit for the data.

Table 1. Descriptive Statistics for the Datasets Used.

\subsection{Acute Myelogeneous Leukemia}

The first real data set represents the survival times, in weeks, of 33 patients suffering from acute Myelogeneous Leukemia. These data have been analyzed by [6]. The data are: $65,156,100,134,16,108,121,4,39,143,56,26,22,1,1,5,65,56,65$, $17,7,16,22,3,4,2,3,8,4,3,30,4,43$. For these data, we shall compare the proposed GGBIII distribution to Gamma Dagum (GD) ([3]), alternative Gamma Dagum (GD) ([11]), Zografos and Balakrishnan Dagum (ZB-D) ([28]).

The asymptotic covariance matrix of the MLEs of the GGBIII model parameters, which is the inverse of the observed Fisher information matrix $I_{n}^{-1}(\widehat{\Delta})$ is given by: 


$$
=\left(\begin{array}{ccccc}
0.0046209593 & 0.0106779546 & -0.000327988 & 2.427039 \mathrm{e}-04 & 12.26055684 \\
0.0106779546 & 0.0246161742 & -0.000759203 & 4.654929 \mathrm{e}-04 & 25.15292231 \\
-0.00032799 & -0.000759203 & 0.0000231093 & -5.77747 \mathrm{e}-05 & -1.55199388 \\
0.0002427039 & 0.0004654929 & -0.000057774 & 1.899781 \mathrm{e}-07 & 0.014450710 \\
12.260556843 & 25.152922308 & -1.551993882 & 1.445071 \mathrm{e}-02 & 1166.046687
\end{array}\right)
$$

Table 1 lists the MLEs of the model parameters for GGBIII, GD, RBD, ZBD models, the corresponding standard errors (given in parentheses) and the statistics $A, \mathrm{KS}$ and $W$.

These results show that the GGBIII distribution has the lowest $A^{*}, \mathrm{KS}$ and $W^{*}$. values among all the fitted models, and so it could be chosen as the best model.

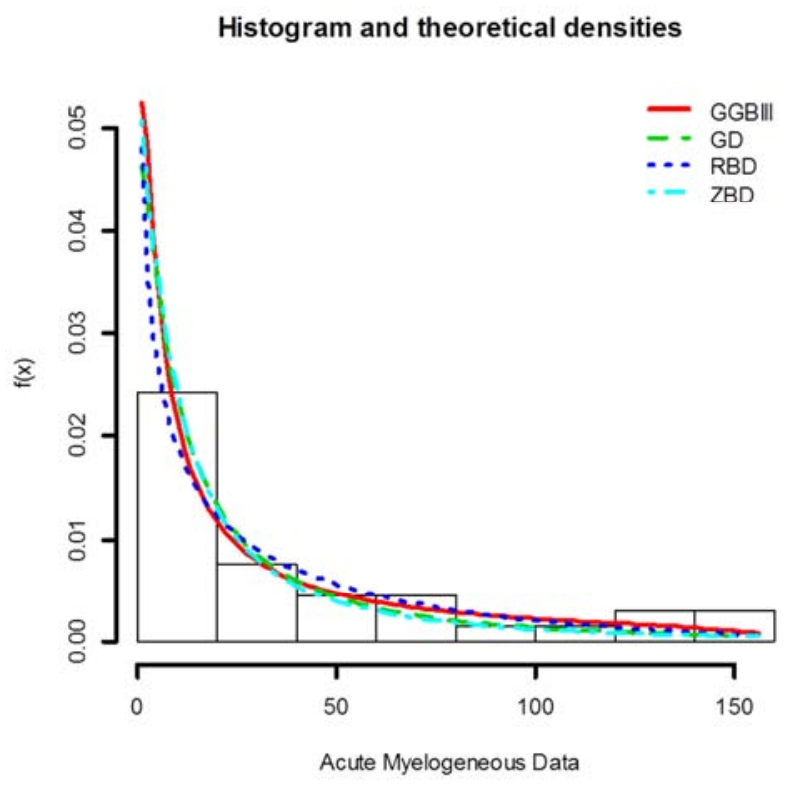

Empirical and theoretical CDFs

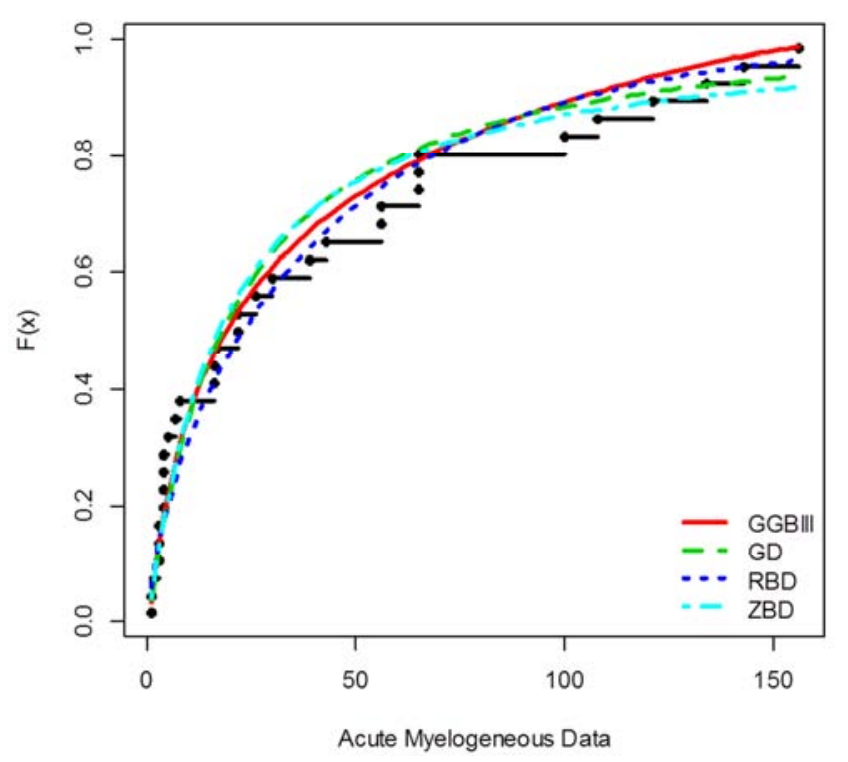

Figure 4. The ECF of the acute myelogeneous data and the estimated fitted distributions.

Figure 3. The histogram of the acute myelogeneous data and the estimated fitted distributions.

Table 2. The Maximum Likelihood Estimation of the Generalized Gamma Burr III distribution for the Acute Myelogeneous Data.

\begin{tabular}{|c|c|c|c|c|c|c|}
\hline MODEL & $\alpha$ & $\beta$ & $\lambda$ & $\delta$ & $p$ & $\theta$ \\
\hline GGBIII & $\begin{array}{c}0.0220 \\
(0.0211)\end{array}$ & $\begin{array}{c}12.510 \\
(12.5082)\end{array}$ & $\begin{array}{l}0.5962 \\
(1.455)\end{array}$ & $\begin{array}{c}0.5817 \\
(0.15417)\end{array}$ & $\begin{array}{l}22.09 \\
(19.3)\end{array}$ & - \\
\hline GD & $\begin{array}{l}0.1663 \\
(0.073)\end{array}$ & $\begin{array}{l}20.1665 \\
(3.1281)\end{array}$ & $\begin{array}{c}5.1135 \\
(1.5994)\end{array}$ & $\begin{array}{c}0.494 \\
(0.0252)\end{array}$ & (1) & $\begin{array}{c}0.001 \\
(0.0005)\end{array}$ \\
\hline ZB-D & $\begin{array}{l}24.167 \\
(0.008)\end{array}$ & $\begin{array}{c}0.00565 \\
(0.01492)\end{array}$ & $\begin{array}{c}0.0004 \\
(0.0013)\end{array}$ & $\begin{array}{c}3.1276 \\
(0.1875)\end{array}$ & - & $(0.0000)$ \\
\hline RBD & $\begin{array}{c}36.585 \\
(223.70) \\
\end{array}$ & $\begin{array}{c}17.7768 \\
(118.129) \\
\end{array}$ & $\begin{array}{l}14.09 \\
(69.47)\end{array}$ & $\begin{array}{c}0.7687 \\
(0.0615) \\
\end{array}$ & - & - \\
\hline
\end{tabular}

Table 3. THE AIC, AICc and BIC for the Distributions.

\begin{tabular}{lllll}
\hline MODEL & -2Log - Likelihood & AIC & AICC & BIC \\
\hline GGBIII & 299.2 & 309.2 & 311.4 & 316.7 \\
GD & 303.6 & 313.6 & 315.8 & 321.1 \\
ZB-D & 308.7 & 316.7 & 318.2 & 322.7 \\
RBD & 307.4 & 315.4 & 316.8 & 321.4 \\
\hline
\end{tabular}

Table 4. Likelihood Ratio Test Statistic.

\begin{tabular}{lll}
\hline MODEL & Hypothesis & LR Statistic \\
\hline GGBIII vs GD & $H_{0}: G D$ vs $H_{1}: G G B I I I$ & 4.4 \\
GGBIII vs RBD & $H_{0}: R B D$ vs $H_{1}:$ GGBIII & 8.2 \\
GGBIII vs ZB-D & $H_{0}: Z B D$ vs $H_{1}: G G B I I I$ & 9.5 \\
\hline
\end{tabular}




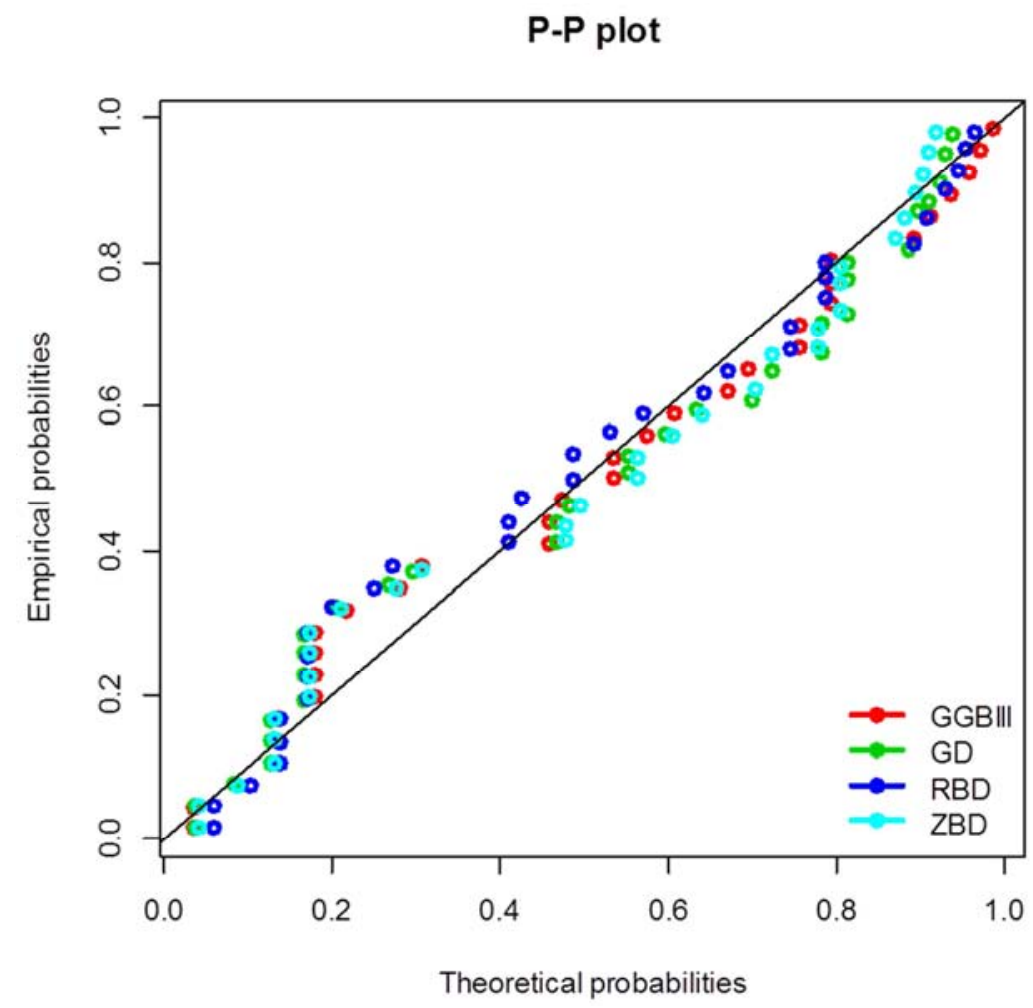

Figure 5. The P-P of the acute myelogeneous data and the estimated fitted distributions.

\subsection{The Air Conditioning System Data}

The second example consists of the number of successive failures for the Air conditioning system of each member in a fleet of 13 Boeing 720 jet airplanes ([22]).

Table 5. Air conditioning system data.

194413907455239750359501304875710215141057320261514492544933318209415860485687111021251414293718629104
7472270283761100615022201201412260335985410011181654912239141839312532943843134184203861827180188230152
53679593324617932720184271562116881301411844154210646230265915310420206566342926355823111832612543634
182512031221821613967310346210577614111976239307441163232223141813341618130901632081247016101522089562
111911471

The asymptotic covariance matrix of the MLEs of the GGBIII model parameters, which is the inverse of the observed Fisher information matrix $I_{n}^{-1}(\widehat{\Delta})$ is given by:

$$
=\left(\begin{array}{ccccc}
0.554348211 & 0.373214023 & -0.0199769930 & 0.0080488570 & 0.272517683 \\
0.373214023 & 0.232368967 & -0.015293134 & 0.0046513590 & 0.151946115 \\
-0.01997699 & -0.01529313 & 0.00045638670 & -0.000727357 & -0.08481367 \\
0.008048857 & 0.004651359 & -0.0007273570 & 6.844605 \mathrm{e}-05 & 0.001974827 \\
0.272517683 & 0.151946115 & -0.0848136726 & 1.974827 \mathrm{e}-03 & 0.051271694
\end{array}\right)
$$

Table 6. The Maximum Likelihood Estimation of the Generalized Gamma Burr III Distribution for the Air Conditioning System Data.

\begin{tabular}{lcccccc}
\hline MODEL & $\boldsymbol{\alpha}$ & $\boldsymbol{\beta}$ & $\boldsymbol{\lambda}$ & $\boldsymbol{\delta}$ & $\boldsymbol{p}$ & $\boldsymbol{\theta}$ \\
\hline \multirow{2}{*}{ GGBIII } & 0.0440 & 9.1609 & 30.8940 & 1.0662 & 3.8490 & - \\
& $(0.0223)$ & $(1.90343)$ & $(13.0829)$ & $(0.0993)$ & $(1.8963)$ & 0.5384 \\
GD & 0.1856 & 31.0783 & 2.1816 & 0.538 & - & $(0.034)$ \\
& $(0.0188)$ & $(7.1966)$ & $(0.8567)$ & $(0.05068)$ & 0.1885 & - \\
ZB-D & 10.6110 & 14.8939 & 0.9507 & $(0.01895)$ & - & - \\
& $(1.9869)$ & $(1.0488)$ & $(0.0375)$ & 0.4739 & - & - \\
RBD & 23.5930 & 9.0448 & 77.9930 & $(0.4477)$ & - & \\
& $(63.608)$ & $(20.2853)$ & $(124.22)$ &
\end{tabular}


Histogram and theoretical densities

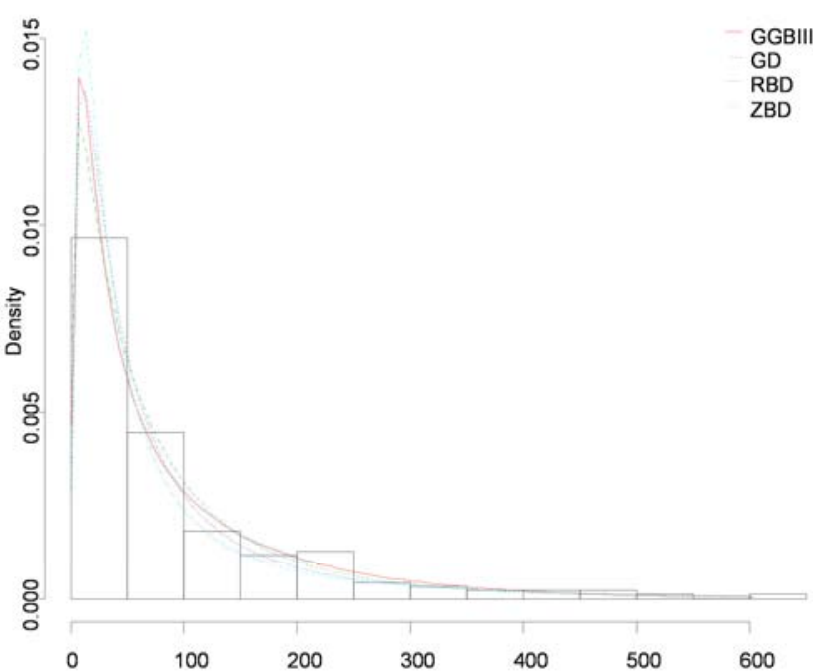

Air Conditioning data

Figure 6. The histogram of the Air conditioning data and the estimated fitted distributions.

\section{Empirical and theoretical CDFs}

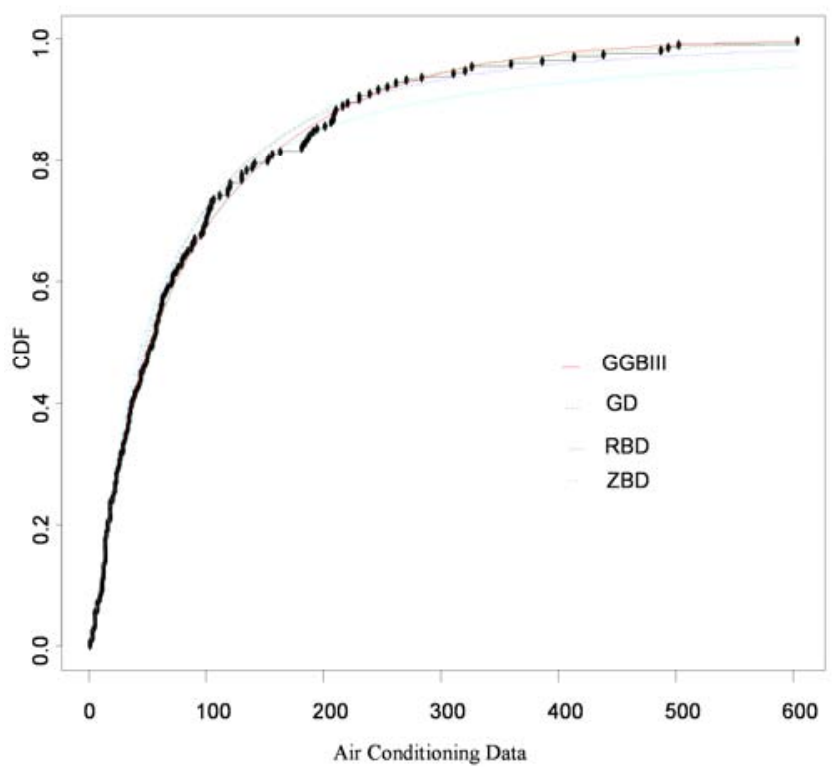

Figure 7. The cumulative function of the Air conditioning data and the estimated fitted distributions.

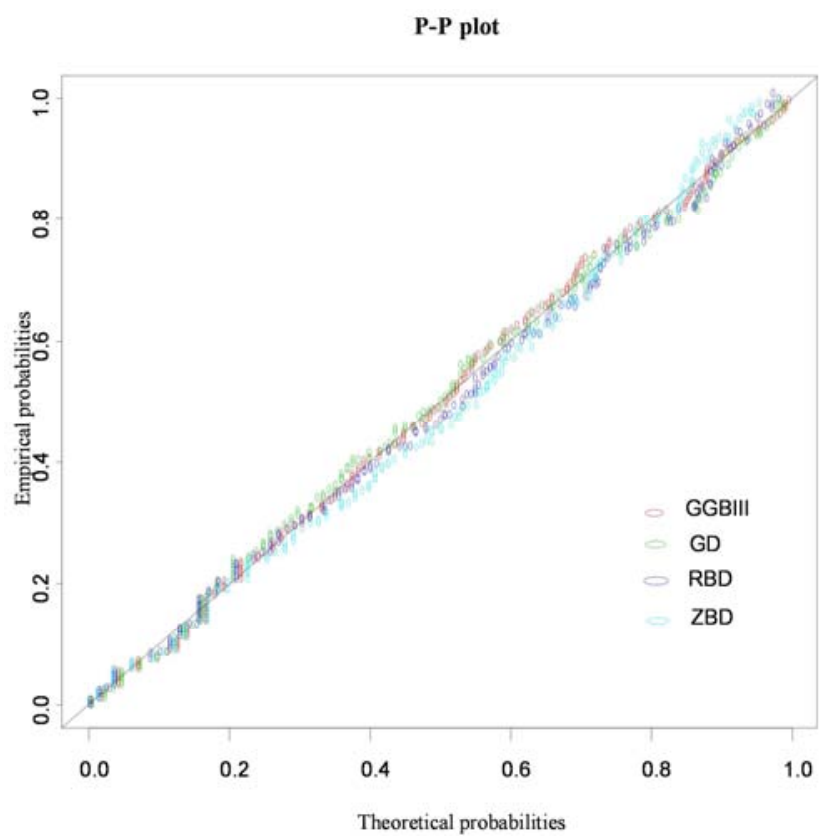

Figure 8. The P-P plot of the Air conditioning data and the estimated fitted distributions.

Table 7. The $-2 L, A I C, A I C c$ and BIC of the Distributions.

\begin{tabular}{lllll}
\hline MODEL & -2LL & AIC & AICC & BIC \\
\hline GGBIII & 2062.9 & 2072.9 & 2073.3 & 2089.1 \\
GD & 2065.1 & 2075.1 & 2075.4 & 2091.2 \\
ZB-D & 2084.7 & 2092.7 & 2092.9 & 2105.7 \\
RBD & 2066.9 & 2074.9 & 2075.1 & 2087.8 \\
\hline
\end{tabular}

Table 8. The Likelihood Ratio Test Statistic.

\begin{tabular}{lll}
\hline MODEL & Hypothesis & LR Statistic \\
\hline GGBIII vs GD & $H_{0}: G D$ vs $H_{1}: G G B I I I$ & 2.2 \\
GGBIII vs RBD & $H_{0}: G D$ vs $H_{1}: G G B I I I$ & 4.0 \\
GGBIII vs ZB-D & $H_{0}: G D$ vs $H_{1}: G G B I I I$ & 21.8 \\
\hline
\end{tabular}

Table 9. Goodness of Fit Statistic for Air Conditioning System Data.

\begin{tabular}{llll}
\hline MODEL & $\begin{array}{c}\text { Cramer-Von } \\
\text { Mises Statistic }\end{array}$ & $\begin{array}{c}\text { Anderson-darling } \\
\text { Statistic }\end{array}$ & $\begin{array}{c}\text { Kolmogorov } \\
\text { Smirnov Statistic }\end{array}$ \\
\hline GGBIII & 0.03536 & 0.26363 & 0.03815 \\
GD & 0.04063 & 0.28902 & 0.04296 \\
RB-D & 0.03807 & 0.30803 & 0.04151 \\
ZBD & 0.13285 & 1.08089 & 0.05574 \\
\hline
\end{tabular}

\subsection{Failure Times of 50 Components (Per 1000 Hours)}

Real dataset was used to show that the GGBIII distribution is a better model when compared to GD, RBD, and ZBD distribution. The dataset taken from ([20]) represents the failure times of 50 components (per 1000h):

Table 10. The Failure Time of 50 Components Data.

\begin{abstract}
$0.036,0.058,0.061,0.074,0.078,0.086,0.102,0.103,0.114,0.116,0.148,0.183,0.192,0.254,0.262,0.379,0.381,0.538,0.570,0.574,0.590,0.618$ $0.645,0.961,1.228,1.600,2.006,2.054,2.804,3.058,3.076,3.147,3.625,3.704,3.931,4.073,4.393,4.534,4.893,6.274,6.816,7.896,7.904,8.022$, $9.337,10.940,11.020,13.880,14.730,15.080$.
\end{abstract}

The asymptotic covariance matrix of the MLEs of the GGBIII model parameters, which is the inverse of the observed Fisher information matrix $I_{n}^{-1}(\widehat{\Delta})$ is given by 


$$
=\left(\begin{array}{ccccc}
1.028647 \mathrm{e}-01 & 8.474526 \mathrm{e}-03 & 0.01401143509 .055202 \mathrm{e}-04 & 12.591021456 \\
8.474526 \mathrm{e}-03 & 3.678572 \mathrm{e}-03 & 0.00513544788 .182118 \mathrm{e}-05 & 1.678922408 \\
1.401144 \mathrm{e}-02 & 5.135448 \mathrm{e}-03 & 0.00492826201 .009937 \mathrm{e}-04 & 2.731526626 \\
9.055202 \mathrm{e}-04 & 8.182118 \mathrm{e}-05 & 0.00010099379 .044504 \mathrm{e}-08 & 0.001921215 \\
1.259102 \mathrm{e}+01 & 1.678922 \mathrm{e}+00 & 2.73152662581 .921215 \mathrm{e}-03 & 43.107069967
\end{array}\right)
$$

Table 11. The Maximum Likelihood Estimation of the Parameters for Failure Time of Components Per Hour.

\begin{tabular}{|c|c|c|c|c|c|c|}
\hline MODEL & $\alpha$ & $\boldsymbol{\beta}$ & $\lambda$ & $\delta$ & $p$ & $\boldsymbol{\theta}$ \\
\hline GGBIII & $\begin{array}{c}0.089 \\
(0.067)\end{array}$ & $\begin{array}{c}4.079 \\
(2.932)\end{array}$ & $\begin{array}{c}0.358 \\
(0.752)\end{array}$ & $\begin{array}{c}0.563 \\
(0.179)\end{array}$ & $\begin{array}{c}5.618 \\
(3.076)\end{array}$ & - \\
\hline GD & $\begin{array}{c}0.560 \\
(0.852)\end{array}$ & $\begin{array}{c}3.838 \\
(3.722)\end{array}$ & $\begin{array}{c}1.681 \\
(1.299)\end{array}$ & $\begin{array}{c}0.417 \\
(0.236)\end{array}$ & (0.0) & $\begin{array}{c}0.104 \\
(0.198)\end{array}$ \\
\hline RB-D & $\begin{array}{c}6.170 \\
(7.756)\end{array}$ & $\begin{array}{c}2.774 \\
(4.290)\end{array}$ & $\begin{array}{c}8.018 \\
(12.910)\end{array}$ & $\begin{array}{c}0.562 \\
(0.483)\end{array}$ & - & - \\
\hline ZB-D & $\begin{array}{c}0.076 \\
(0.011)\end{array}$ & $\begin{array}{c}5.424 \\
(0.004)\end{array}$ & $\begin{array}{l}7.930 \\
(0.019)\end{array}$ & $\begin{array}{c}1.331 \\
(0.004)\end{array}$ & - & - \\
\hline
\end{tabular}

Table 12. The $-2 L$, AIC and BIC of the Distributions for Failure Time of Components per Hour.

\begin{tabular}{llll}
\hline MODEL & $\mathbf{- 2 L o g}$ - Likelihood & AIC & BIC \\
\hline GGBIII & 198.4 & 208.4 & 217.9 \\
GD & 205.9 & 215.9 & 255.4 \\
ZB-D & 207.9 & 215.9 & 223.6 \\
RBD & 205.5 & 213.5 & 221.2 \\
\hline
\end{tabular}

Table 13. The Likelihood Ratio Test Statistic.

\begin{tabular}{lll}
\hline MODEL & Hypothesis & LR Statistic \\
\hline GGBIII vs GD & $H_{0}:$ GD vs $H_{1}: G G B I I I$ & 7.5 \\
GGBIII vs RBD & $H_{0}:$ GD vs $H_{1}: G G B I I I$ & 7.1 \\
GGBIII vs ZB-D & $H_{0}:$ GD vs $H_{1}: G G B I I I$ & 9.5 \\
\hline
\end{tabular}

Table 14. Goodness of Fit Statistic for Components Data.

\begin{tabular}{lllc}
\hline \multirow{2}{*}{ MODEL } & $\begin{array}{c}\text { Cramer - Von } \\
\text { Mises Statistic }\end{array}$ & $\begin{array}{c}\text { Anderson-darling } \\
\text { Statistic }\end{array}$ & 0.10417 \\
\hline GGBIII & 0.12097 & 0.75666 & 0.12379 \\
GD & 0.16636 & 1.02411 & 0.12923 \\
RB-D & 0.17121 & 1.05099 & 0.11922 \\
ZB-D & 0.14148 & 0.92404 & Statistic \\
\hline
\end{tabular}

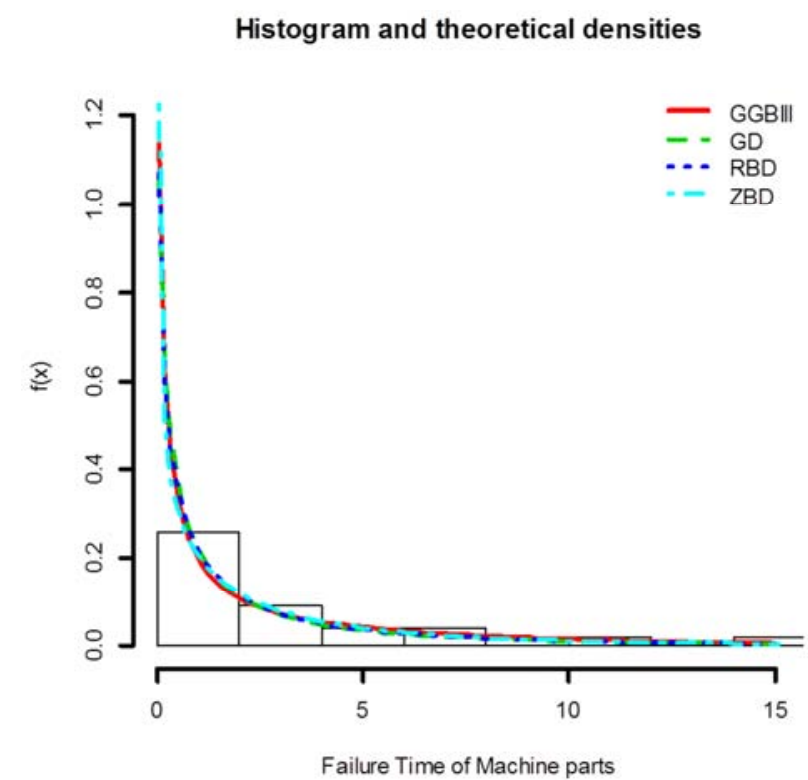

Figure 9. The histogram of the Air conditioning data and the estimated fitted distributions.
Empirical and theoretical CDFs

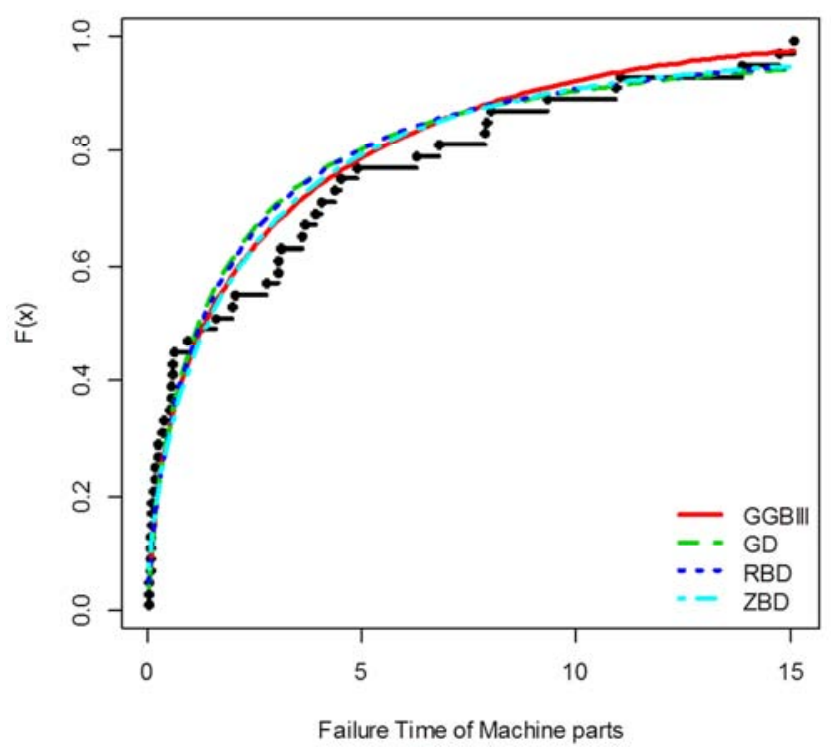

Figure 10. The Ecf of the Air conditioning data and the estimated fitted distributions. 


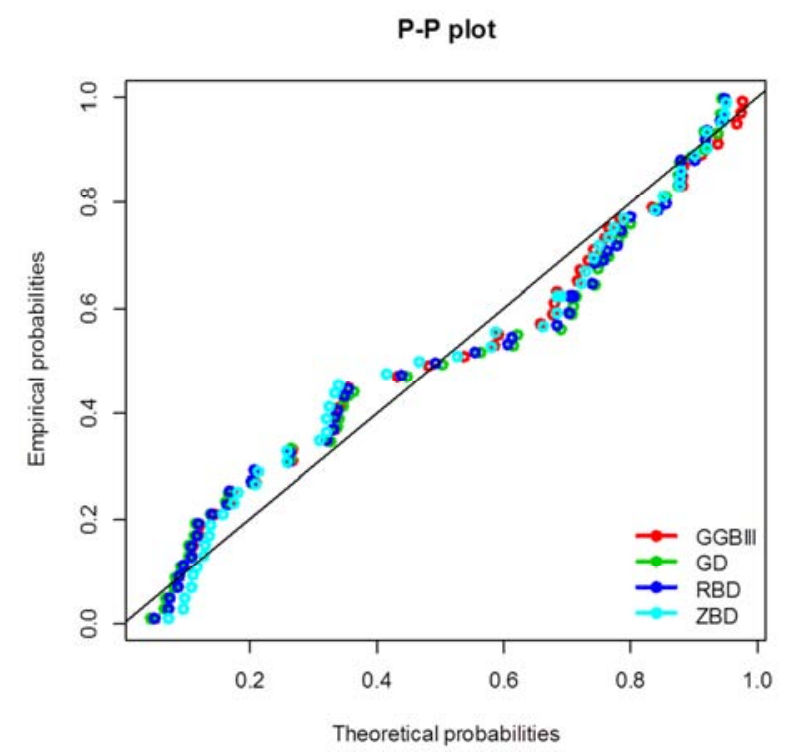

Figure 11. The P-P plot of the Air conditioning data and the estimated fitted distributions.

Empirical survival function for FAILURE MACHINE data

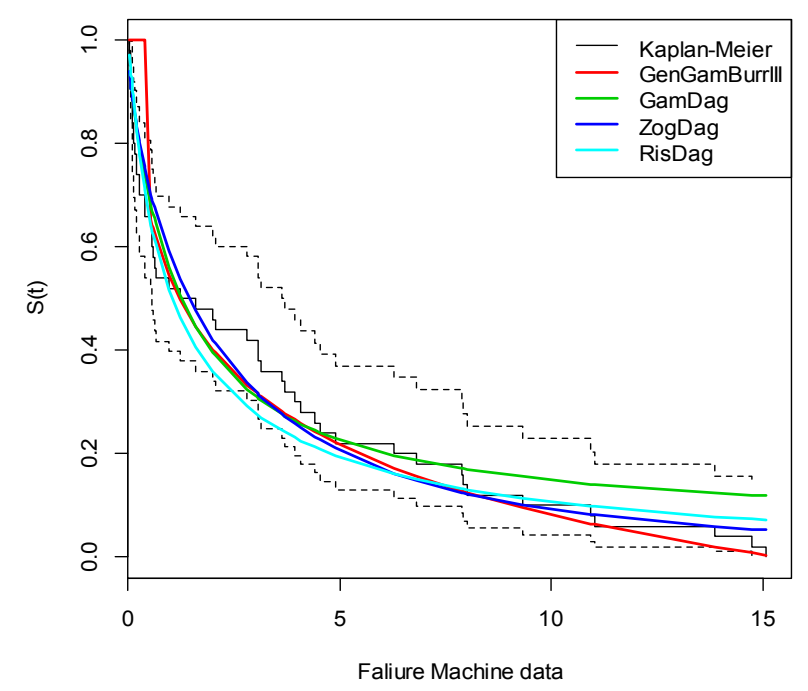

Figure 12. The Esf of the Air conditioning data and the estimated fitted distributions.

\subsection{Breast Cancer Survival Data}

The application of the GGBII distribution to a breast cancer data was developed. The study cohort comprises 1207 patients with cancer treated by mastectomy. Patient data were obtained from the database of SPSS software. The data consist of number of months after mastectomy. Uncensored observations correspond to patients having death time computed. Censored observations correspond to patients who were not observed to have died at the time the data were collected. The numbers of censored and uncensored observations are 1135 and 72, respectively, of the total of 1207 patients.

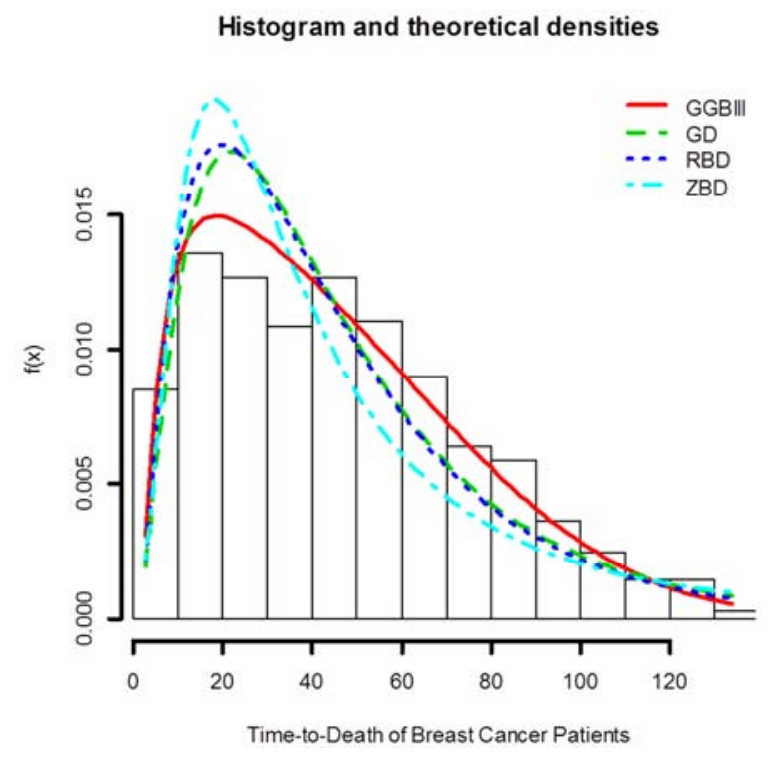

Figure 13. The histogram of the Breast cancer data and the estimated fitted distributions.

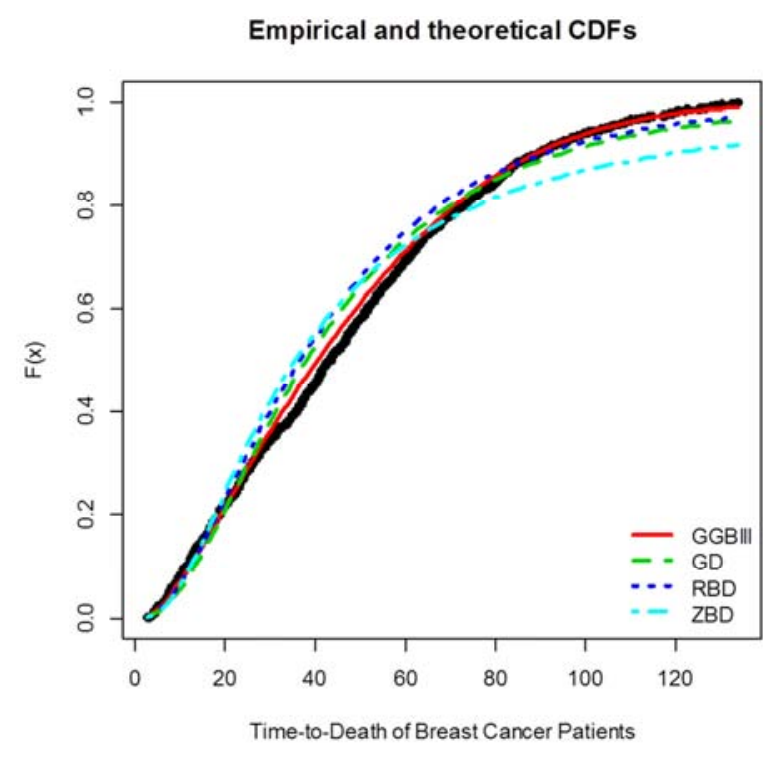

Figure 14. The Ecf of the Breast cancer data and the estimated fitted distributions.

The asymptotic covariance matrix of the MLEs of the GGBIII model parameters, which is the inverse of the observed Fisher information matrix $I_{n}^{-1}(\widehat{\Delta})$ is given by:

$$
=\left(\begin{array}{ccccc}
0.027479129 & 0.0070660643 & -1.08292 \mathrm{e}-03 & 1.209875 \mathrm{e}-03 & 0.0932442544 \\
0.007066064 & 0.0017795919 & -2.87801 \mathrm{e}-042.736553 \mathrm{e}-04 & 0.0193527713 \\
-0.0010829 & -0.000287801 & 3.732178 \mathrm{e}-05-1.02690 \mathrm{e}-04 & 0.1099804175 \\
0.001209875 & 0.0002736553 & -1.02690 \mathrm{e}-041.710644 \mathrm{e}-05 & 0.0008351013 \\
0.093244254 & 0.0193527713 & 1.099804 \mathrm{e}-018.351013 \mathrm{e}-04 & 0.0354401755
\end{array}\right)
$$


Table 15. The Maximum Likelihood Estimation of the Parameters for Time-to-Death of the Breast Cancer Patients.

\begin{tabular}{|c|c|c|c|c|c|c|}
\hline MODEL & $\alpha$ & $\beta$ & $\lambda$ & $\delta$ & $p$ & $\boldsymbol{\theta}$ \\
\hline GGBIII & $\begin{array}{c}0.2523 \\
(0.0363)\end{array}$ & $\begin{array}{l}10.1887 \\
(2.3334)\end{array}$ & $\begin{array}{c}0.8490 \\
(0.3655)\end{array}$ & $\begin{array}{c}0.6490 \\
(0.0456)\end{array}$ & $\begin{array}{c}6.0770 \\
(0.5019)\end{array}$ & \\
\hline GD & $\begin{array}{c}0.4417 \\
(0.0363)\end{array}$ & $\begin{array}{c}32.1491 \\
(2.1059)\end{array}$ & $\begin{array}{c}1.3056 \\
(0.0925)\end{array}$ & $\begin{array}{c}0.5710 \\
(0.0233)\end{array}$ & - & $\begin{array}{c}0.030 \\
(0.0097)\end{array}$ \\
\hline ZB-D & $\begin{array}{l}0.17549 \\
(0.0052)\end{array}$ & $\begin{array}{l}12.0983 \\
(0.0097)\end{array}$ & $\begin{array}{c}80.292 \\
(0.01515)\end{array}$ & $\begin{array}{c}1.4590 \\
(0.0045)\end{array}$ & & \\
\hline RBD & $\begin{array}{c}14.3930 \\
(11.7487)\end{array}$ & $\begin{array}{c}5.5995 \\
(3.5564)\end{array}$ & $\begin{array}{c}370.23 \\
(190.1162) \\
\end{array}$ & $\begin{array}{c}0.9590 \\
(0.2591) \\
\end{array}$ & & \\
\hline
\end{tabular}

Table 16. THE -2L, AIC, AICC AND BIC OF THE MODELS.

\begin{tabular}{llll}
\hline MODEL & $\mathbf{- 2 L o g}$ - Likelihood & AIC & BIC \\
\hline GGBIII & 11332.4 & 11342.4 & 11367.9 \\
GD & 11439.7 & 11449.7 & 11475.2 \\
ZB-D & 11640.8 & 11650.8 & 11671.2 \\
RBD & 11454.4 & 11464.4 & 11484.8 \\
\hline
\end{tabular}

Table 17. The Likelihood Ratio Test Statistic.

\begin{tabular}{lll}
\hline ODEL & Hypothesis & LR Statistic \\
\hline GGBIII vs. GD & $H_{0}:$ GD vs $H_{1}: G G B I I I$ & 107.33 \\
GGBIII vs. RBD & $H_{0}: G D$ vs $H_{1}: G G B I I I$ & 122.01 \\
GGBIII vs. ZB-D & $H_{0}: G D$ vs $H_{1}: G G B I I I$ & 308.44 \\
\hline
\end{tabular}

Table 18. Goodness of Fit Statistic for Breast Cancer Data.

\begin{tabular}{llll}
\hline MODEL & $\begin{array}{c}\text { Cramer-Von } \\
\text { Mises Statistic }\end{array}$ & $\begin{array}{c}\text { Kolmogorov } \\
\text { Smirnov Statistic }\end{array}$ \\
\hline GGBIII & 0.44366 & 2.18208 & 0.03976 \\
GD & 3.05180 & 14.78988 & 0.09135 \\
RB-D & 1.86892 & 10.95541 & 0.07256 \\
ZB-D & 3.86745 & 23.31508 & 0.10140 \\
\hline
\end{tabular}

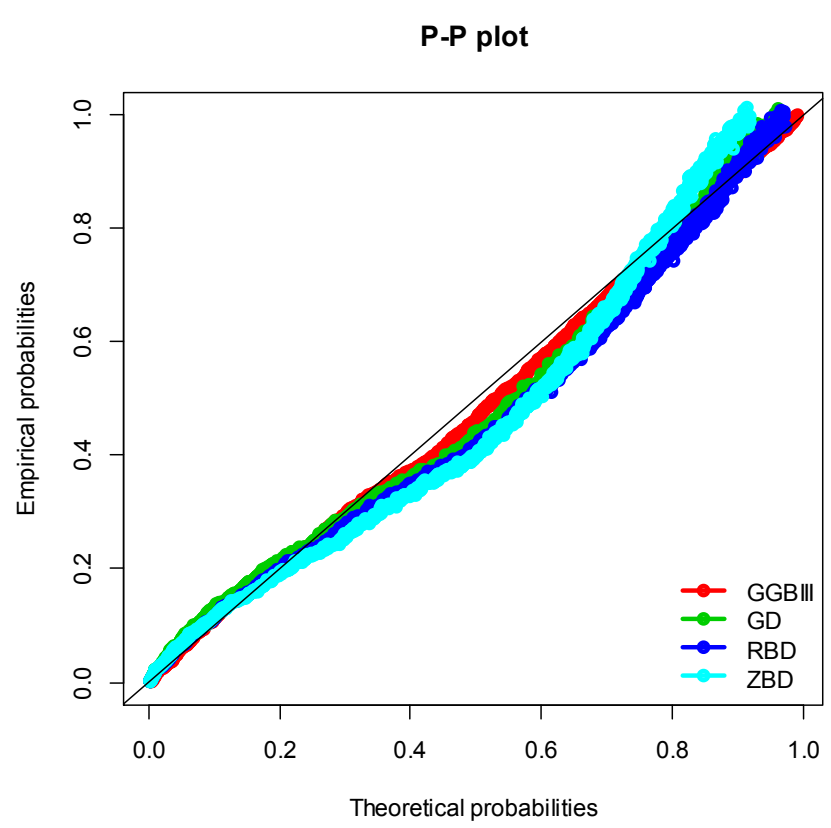

Figure 15. The P-P plot of the Breast cancer data and the estimated fitted distributions.

Summary of the Findings

A new five-parameter distribution named the Generalized Gamma Burr III distribution has been introduced. It is the generalization of the Burr III distribution.

The proposed distribution has the ability to capture monotonically increasing, decreasing and unimodal hazard rates.

It also reveals that GGBIII distribution has widened the scope of gamma-G family into the area of survival analysis and it has been found amenable in the medical area.

Finally, it was shown that the proposed distribution gave the best fit for five well-known data sets (when compared to other distributions including one having five parameters).

\section{Discussion}

A new class of generalized Burr III distribution called the generalized gamma-Burr III distribution is proposed and studied. The idea is to combine two components in a serial system, so that the hazard function is either increasing or more importantly bathtub shaped and unimodal shaped. The GGBIII distribution has the family of Zografos and Balakrishnan distribution as special cases. The density of this new class of distributions was expressed as a linear combination of Burr III density functions. The GGBIII distribution possesses hazard function with flexible behavior. Also obtained was a closed form expressions for the moments, mean and median deviations, and distribution of order statistics. Maximum likelihood estimation technique was used to estimate the model parameters.

Moreover, to have a strong evidence for this work, the goodness of fit plot for each dataset was provided in order to check how fit the proposed model is to the dataset as compared to other models. Finally, the GGBIII model was applied to FOUR different types of real datasets to illustrate 
the usefulness and robustness of the distribution in different areas including medical areas and also the model outperformed the existing models of Gamma-Generated

Family and is found better than GD and ZBD and RBD which have been fitted to the data used except the breast cancer data. Also, the new model was specifically applied to censored data and it was found more flexible.

\section{Conclusion}

This paper introduced for the first time the usefulness of the new distribution in survival analysis aside finance, economic, and reliability studies, and it was found that there is a wide significant different in the breast cancer data analysis that is the new distribution is useful in survival analysis far more than other generalizations through Gamma generated family. Thus, we conclude that GENERALIZED GAMMA BURR III is an alternative distribution to gamma families.

\section{References}

[1] Al-Dayian, G. R., 1999. Burr type III distribution: Properties and Estimation. The Egyptian Statistical Journal, 43: 102-116.

[2] Antonio, E. G. and da-Silva, C. Q 2014. The Beta Burr III Model for Lifetime Data.

[3] Broderick, O. O., Shujiao, H. and Mavis, P. 2014. A New Class of Generalized Dagum Distribution with Applications to Income and Lifetime Data. Journal of Statistical and Econometric Methods, vol. 3, no. 2.

[4] Burr, I. W., 1942. Cumulative frequency distributions. Annals of Mathematical Statistics, 13, 215-232.

[5] Eugene, N., Lee, C., and Famoye, F. 2002. Beta-Normal distribution and its application. Communication in Statistics Theory and Methods, 4: pp. 497-512.

[6] Feigl, P., and Zelen, M. 1965. Estimation of exponential probabilities with concomitant information. Biometrics, 21: 826-838.

[7] Gove, J. H., Ducey M. J., Leak, W. B. and Zhang, L., 2008. Rotated sigmoid structures in managed uneven-aged northern hardwood stands: a look at the Burr type III distribution. Forestry, (5 February 2008).

[8] Gradshteyn, I. S. and Ryzhik, I. M. 2000. Table of Integrals, Series, And Products, Seventh Edition.

[9] Gupta, R. D. and Kundu, D., 1999. Generalized exponential distributions. Austral and New Zealand J. Statist., 41 (2): 173188.

[10] Hose, G. C. 2005. Assessing the Need for Groundwater Quality Guidelines for Pesticides Using the Species Sensitivity Distribution Approach. Human and Ecological Risk Assessment, 11: 951-966.

[11] Jailson, A. R. and Ana P. C. 2015. The Gamma-Dagum Distribution: Definition, Properties And Application. Vol 3, No:1.
[12] Jones, M. C. 2004. Families of distributions arising from distributions of order statistics. TEST, 13: 1-43.

[13] Klugman, S. A., Panjer, H. H. and Willmot, G. E., 1998. Loss Models. John Wiley, New York.

[14] Lindsay, S. R., Wood, G. R. and Woollons, R. C. 1996. Modeling the diameter distribution of forest stands using the Burr distribution. Journal of Applied Statistics, 23: 609-619.

[15] Luis, B. P., Cordeiro, G. M. and Juvencio S. N. 2012. The Gamma-Exponentiated Weibull Distribution. Journal of Statistical Theory and Applications Volume 11, Number 4, pp. 379-395.

[16] Marcelino, P., Ortega, E. M. and Cordeiro, G. M. 2011. The Kumaraswamy generalized gamma distribution with application in survival analysis. Statistical methodology 8.5: 411-433.

[17] Mielke, P. W. 1973. Another family of distributions for describing and analyzing precipitation data. Journal of Applied Meteorology, 12: 275-280.

[18] Mielke, P. W., Johnson, E. S., 1973. Three-parameter kappa distribution maximum likelihood estimates and likelihood ratio test. Monthly Weather Review, 101: 701-707.

[19] Mokhlis, N. A. 2005. Reliability of a Stress-Strength Model with Burr type III Distributions. Communications in Statistics - Theory and Methods, 34: 1643-1657.

[20] Murthy, D. P., Xie, M., and Jiang, R. 2004. Weibull models (Vol. 505). John Wiley and Sons.

[21] Ojo, M. O. and Olapade, A. K. 2005. On a generalization of the Pareto distribution, Proceedings of the International Conference in honor of Prof. E. O. Oshobi and Dr. J. O. Amao, 65-70.

[22] Proschan, F. 1963. Theoretical Explanation of Observed Decreasing Failure Rate, Technometrics, 5: 375-383.

[23] Ristic, M. and Balakrishnan, N. 2012. The gammaexponentiated exponential distribution. J. Stat. Comp. Simulation, 82 (8): 1191-1206.

[24] Ristic, M. and Balakrishnan, N. 2011. The gammaexponentiated exponential distribution. J. Statist. Comput. Simulation.

[25] Shao, Q., Chen, Y. D., and Zhang, L., 2008. An extension of three-parameter Burr III distribution for low-flow frequency analysis. Computational Statistics and Data Analysis, 52: 1304-1314.

[26] Shao, Q., 2000. Estimation for hazardous concentrations based on NOEC toxicity data: an alternative approach. Environmetrics, 11: 583-595.

[27] Sherrick, B. J., Garcia, P., and Tirupattur, V. 1996. Recovering probabilistic information from option markets: test of distributional assumptions. The Journal of Future Markets, 16: $545-560$.

[28] Zografos, K. and Balakrishnan, N. 2009. On Families of betaand Generalized Gamma-Generated Distribution and Associated Inference, Stat. Method, 6: 344-362. 